\title{
छs \\ Electromagnetic forces on plasmonic nanoparticles induced by fast electron beams
}

\author{
Alejandro Reyes-Coronado* \\ Donostia International Physics Center, Paseo Manuel Lardizabal 4, Donostia-San Sebastián 20018, Spain \\ and Institute of Electronic Structure and Laser (IESL), Foundation for Research and Technology Hellas (FORTH), \\ P.O. Box 1385, 71110 Heraklion, Crete, Greece \\ Rubén G. Barrera \\ Instituto de Física, Universidad Nacional Autónoma de México, Apartado Postal 20-364, 01000 México D.F., Mexico \\ Philip E. Batson \\ IBM Thomas J. Watson Research Center, Yorktown Heights, New York 10598, USA \\ and Institute for Advanced Materials, Devices, and Nanotechnology, Rutgers University, 607 Taylor Road, \\ Piscataway, New Jersey 08854, USA \\ Pedro M. Echenique and Alberto Rivacoba \\ Department of Materials Physics, Universidad del Pais Vasco (UPV)/EHU, \\ Paseo Manuel Lardizabal 4, Donostia-San Sebastián 20018, Spain \\ and Centro de Física de Materiales, CSIC-UPV/EHU and Donostia International Physics Center, DIPC, \\ Paseo Manuel Lardizabal 5, Donostia-San Sebastián 20018, Spain \\ Javier Aizpurua ${ }^{\dagger}$ \\ Centro de Física de Materiales, CSIC-UPV/EHU and Donostia International Physics Center, DIPC, \\ Paseo Manuel Lardizabal 5, Donostia-San Sebastián 20018, Spain
}

(Received 11 August 2010; published 15 December 2010)

\begin{abstract}
The total momentum transfer from fast electron beams, like those employed in scanning transmission electron microscopy (STEM), to plasmonic nanoparticles is calculated. The momentum transfer is obtained by integrating the electromagnetic forces acting on the particles over time. Numerical results for single and dimer metallic nanoparticles are presented, for sizes ranging between 2 and $80 \mathrm{~nm}$. We analyze the momentum transfer in the case of metallic dimers where the different relevant parameters such as particle size, interparticle distance, and electron beam impact parameter are modified. It is shown that depending on the specific values of the parameters, the total momentum transfer yields a force that can be either attractive or repulsive. The time-average forces calculated for electron beams commonly employed in STEM are on the order of piconewtons, comparable in magnitude to optical forces and are thus capable of producing movement in the nanoparticles. This effect can be exploited in mechanical control of nanoparticle induced motion.
\end{abstract}

DOI: $10.1103 /$ PhysRevB.82.235429

PACS number(s): 73.20.Mf, 68.37.Ma, 42.50.Wk, 41.75.Fr

\section{INTRODUCTION}

Electrons have been used since the middle of the last century as a probe to obtain information on the nature and properties of condensed and soft matter. Scanning transmission electron microscope (STEM) has been shown to be an efficient tool to probe electronic excitations in structures at nanometric scales. ${ }^{1-8}$ Nowadays, modern electron microscopes achieve atomic resolution, with high electron energies between 200 and $300 \mathrm{keV}$ and very well focused beams, down to $0.8-0.9 \AA$, renewing and stimulating the interest in the interaction of high-energy electron beams with interfaces and particles. ${ }^{9-12} \mathrm{~A}$ recent and novel observation shows that STEM can induce motion in clusters of metallic nanoparticles. ${ }^{13}$ Electrons couple electromagnetically to the particles, inducing surface-charge-density excitations, known as surface plasmons, ${ }^{14,15}$ producing local fields that transfer momentum to the particles. In the case of a single particle, this momentum transfer depends on the impact parameter of the electron beam, on the size of the nanoparticle, and on the dielectric response of the material the particle is made of. ${ }^{16}$ In the case of a dimer, in addition to the interaction between the surface-charge-density excitations induced on the particle and the electron, the interaction between the two particles plays an important role, generating even more localized plasmons between the particles ${ }^{17-20}$ which are a consequence of the hybridization of the single-particle modes. ${ }^{21}$ These hybridized modes are responsible for the electromagnetic field enhancement present in the interparticle junction. For very short separation distances between particles, this field enhancement can be extremely large $\mathrm{e}^{20,22}$ and consequently the forces acting on the particles become quite strong.

In this work, we present calculations of the total momentum transfer from fast electrons to single and dimer metallic nanoparticles in typical STEM experimental configurations. In the case of a single particle, characterized with a dielectric function $\varepsilon(\omega)$, it is shown that the forces acting on the particle can be attractive, i.e., the particle moves toward the electron trajectory, or repulsive, moving the particle away from the electron trajectory, depending on the specific values 
of particle size and electron impact parameter, in agreement with previous work. ${ }^{16}$ In the case of a metallic dimer, where the separation distance between the particles becomes another relevant parameter, it is shown that for specific values of these parameters the interaction between the electron and the particles can drive the two particles together (we also have observed that the particles can be driven apart for suitable set of parameters). The magnitude of the calculated time-average forces acting on the nanoparticles is comparable in magnitude to the laser-induced forces (on the order of piconewtons) reported in previous work on metallic nanoparticle clusters, both with use of a nonretarded approach ${ }^{23}$ and a full electromagnetic approach. ${ }^{24,25}$

Several factors such as the interaction between the particles and the supporting substrate material, charging effects, heating of the particles, etcetera, can have an effect in the motion of particles under the influence of an electron beam. Nevertheless, we focus here on the mechanism driven by electromagnetic forces induced by fast electron beams through the excitation of localized surface plasmons on the metallic nanoparticles. We show that these forces can be strong enough to drive particles together, yielding coalescence phenomenon as had been recently observed experimentally. ${ }^{13}$

\section{THEORY}

The total momentum transfer $\vec{P}$ from a fast electron to a particle in vacuum can be calculated by adding the contributions to the force produced on the particle along the electron trajectory, ${ }^{26}$ or equivalently integrating over time the instantaneous force $\vec{F}_{\text {mec }}(t)$. Considering the equation for the conservation of momentum ${ }^{27}$ and integrating it over time, the total momentum transfer $\vec{P}$ is given by

$$
\vec{P}=\int_{-\infty}^{\infty} \frac{d}{d t}\left[\vec{P}_{m e c}(t)+\vec{P}_{E M}(t)\right] d t=\int_{-\infty}^{\infty} \oint_{S} \stackrel{\leftrightarrow}{T}(\vec{r} ; t) \cdot \vec{d} a d t,
$$

where $\vec{P}_{m e c}$ is the mechanical momentum, $\vec{P}_{E M}$ is the linear momentum carried by the electromagnetic field, and $\overleftrightarrow{T}(\vec{r} ; t)$ is the Maxwell stress tensor. Here $S$ denotes a closed surface surrounding the particle that experiences the force and $\vec{d} a$ is the differential area vector normal to the surface $S$, pointing outwardly.

The term corresponding to the electromagnetic momentum, $\vec{P}_{E M}$, does not contribute to the total momentum transfer from the electron to the particle. ${ }^{28}$ The total momentum transfer is therefore given by

$$
\vec{P}=\int_{-\infty}^{\infty} \frac{d}{d t} \vec{P}_{m e c}(t) d t=\int_{-\infty}^{\infty} \oint_{S} \overleftrightarrow{T}(\vec{r} ; t) \cdot \vec{d} a d t
$$

We identify now $d / d t \vec{P}_{m e c}(t)$ as the mechanical force $\vec{F}_{m e c}(t)$ and substitute in the first integral of Eq. (2) its Fourier transform. We interchange the order of integration and write

$$
\begin{aligned}
\vec{P} & =\int_{-\infty}^{\infty} \vec{F}_{m e c}(\omega) \int_{-\infty}^{\infty} e^{-i \omega t} d t \frac{d \omega}{2 \pi} \\
& =\int_{-\infty}^{\infty} \vec{F}_{m e c}(\omega) \delta(\omega) d \omega=\vec{F}_{m e c}(\omega=0),
\end{aligned}
$$

where we can observe that the total momentum transfer is given by the $\omega=0$ component of the Fourier transform of the mechanical force. On the other hand, from Eq. (1), the total momentum transfer is given by

$$
\vec{P}=\oint_{S}\left[\int_{-\infty}^{\infty} \stackrel{\leftrightarrow}{T}(\vec{r} ; t) d t\right] \cdot \vec{d} a
$$

Considering the Fourier transform in the time domain of the Maxwell stress tensor, one can identify the time integral of $\stackrel{\leftrightarrow}{T}(\vec{r} ; t)$ inside the square brackets in Eq. (4) as the zerofrequency component of the Maxwell stress tensor, $\stackrel{\leftrightarrow}{T}(\vec{r} ; \omega$ $=0)$. Thus, one can write the expression for the total momentum transfer as follows:

$$
\vec{P}=\vec{F}_{m e c}(\omega=0)=\oint_{S} \stackrel{\leftrightarrow}{T}(\vec{r} ; \omega=0) \cdot \vec{d} a
$$

The Maxwell stress tensor in dyadic form, using SI system of units, ${ }^{27}$ is given by

$$
\begin{aligned}
\overleftrightarrow{T}(\vec{r} ; \omega=0)= & \int_{-\infty}^{\infty} \overleftrightarrow{T}(\vec{r} ; t) d t \\
= & \int_{-\infty}^{\infty}\left[\varepsilon_{0} \vec{E}(\vec{r} ; t) \vec{E}(\vec{r} ; t)-\frac{\varepsilon_{0}}{2} \stackrel{\leftrightarrow}{I} \vec{E}(\vec{r} ; t) \cdot \vec{E}(\vec{r} ; t)\right. \\
& \left.+\mu_{0} \vec{H}(\vec{r} ; t) \vec{H}(\vec{r} ; t)-\frac{\mu_{0}}{2} \stackrel{\leftrightarrow}{H}(\vec{r} ; t) \cdot \vec{H}(\vec{r} ; t)\right] d t
\end{aligned}
$$

where $\vec{E}(\vec{r} ; t)$ is the electric field and $\vec{H}(\vec{r} ; t)$ is the $H$ field. Replacing the electric and $H$ fields by their corresponding Fourier transform, and using that $\vec{E}^{*}(\omega)=\vec{E}(-\omega)$, since $\vec{E}(t)$ is a real function, the first term on the right-hand side of Eq. (6) becomes

$$
\int_{-\infty}^{\infty} \vec{E}(\vec{r} ; t) \vec{E}(\vec{r} ; t) d t=\frac{1}{2 \pi} \int_{-\infty}^{\infty} \vec{E}\left(\vec{r} ; \omega^{\prime}\right) \vec{E}^{*}\left(\vec{r} ; \omega^{\prime}\right) d \omega^{\prime} .
$$

Since $\vec{E}(t)$ is a real-valued function, it is possible to show that the product $\vec{E}(\vec{r} ; \omega) \vec{E}^{*}(\vec{r} ; \omega)$ (same stands for $H$ ) is an even function in $\omega$, thus performing the same procedure over the other three terms on the right-hand side of Eq. (6), we finally obtain

$$
\vec{P}=\int_{0}^{\infty} \frac{d \vec{P}}{d \omega} d \omega
$$

where 


$$
\begin{aligned}
& \frac{d \vec{P}}{d \omega}=\frac{1}{\pi} \oint_{S}\left[\varepsilon_{0} \vec{E}(\vec{r} ; \omega) \vec{E}^{*}(\vec{r} ; \omega)-\frac{\varepsilon_{0}}{2} \stackrel{I}{E}(\vec{r} ; \omega) \cdot \vec{E}^{*}(\vec{r} ; \omega)\right. \\
& \left.+\mu_{0} \vec{H}(\vec{r} ; \omega) \vec{H}^{*}(\vec{r} ; \omega)-\frac{\mu_{0}}{2} \stackrel{\leftrightarrow}{H}(\vec{r} ; \omega) \cdot \vec{H}^{*}(\vec{r} ; \omega)\right] \cdot \vec{d} a .
\end{aligned}
$$

Note that the expression inside the square brackets of Eq. (9) is not the Fourier transform of the Maxwell stress tensor, nor the Fourier transform of the force, it is rather $\overleftrightarrow{T}(\vec{r} ; \omega=0)$. Thus, as shown in Eq. (5), the total momentum transfer corresponds to the $\omega=0$ component of $\overleftrightarrow{T}(\vec{r} ; \omega)$, the time average of the instantaneous momentum transfer. In Eq. (9), $\vec{E}$ and $\vec{H}$ stand for the total electric and magnetic field, which are given by the sum of the external fields $\vec{E}_{e x t}$ and $\vec{H}_{\text {ext }}$ (the ones produced by the bare electron) and the induced fields $\vec{E}_{\text {ind }}$ and $\vec{H}_{\text {ind }}$,

$$
\begin{gathered}
\vec{E}(\vec{r} ; \omega)=\vec{E}_{e x t}(\vec{r} ; \omega)+\vec{E}_{\text {ind }}(\vec{r} ; \omega) \quad \text { and } \\
\vec{H}(\vec{r} ; \omega)=\vec{H}_{e x t}(\vec{r} ; \omega)+\vec{H}_{\text {ind }}(\vec{r} ; \omega) .
\end{gathered}
$$

In a plasmonic particle or dimer, the integration of the total field will give us the total momentum transfer. The type of response of the particle will be responsible of the type of momentum transfer produced. We will focus on metallic particles described by a Drude-type response function, and also on $\mathrm{Au}$ particles where the optical response includes both plasmonic response and interband transitions. In a particle presenting pure plasmonic resonances, the reaction force as the one coming from a simple harmonic oscillator changes sign (phase) as the driving force frequency is shifted through the resonance. The reaction force is in phase below resonance frequency and out of phase above the resonant frequency. This effect will be clear in Drude-type particles, as we will detail below. In more realistic particles such as gold particles, the response of the material can present highfrequency tails that actually govern the characteristics of the force. In this case, it might be more proper to speak of "dielectric" forces rather than purely "plasmonic" forces. We will show both types of particles for completeness.

\section{MOMENTUM TRANSFER OF A FAST ELECTRON TO A SINGLE METALLIC NANOPARTICLE}

First we study the momentum transfer from an electron to a single metallic spherical nanoparticle considering two cases: a metallic nanosphere characterized by a dielectric response function given by the Drude model and a gold metallic nanosphere characterized by an experimental bulk dielectric function. ${ }^{29}$

\section{A. Drude-type nanosphere}

Let us consider the calculation of the total momentum transfer from a fast electron to a single spherical particle in vacuum, characterized by a dielectric response function $\varepsilon(\omega)$ given by the Drude model,


(a)

(b)

FIG. 1. (Color online) Schematic representation of a fast electron moving with speed $v$ and impact parameter $b$ with respect to: (a) single polarizable particle and (b) dimer of polarizable particles separated by a distance $d . \varepsilon_{i n}$ is the dielectric function characterizing each particle and $\varepsilon_{0}$ is the dielectric function of vacuum.

$$
\varepsilon(\omega)=1-\frac{\omega_{p}}{\omega(\omega+i \Gamma)}
$$

with the plasma frequency $\omega_{p}=15.1 \mathrm{eV}$ and damping $\Gamma$ $=0.15 \mathrm{eV}$ (simulating an aluminum particle). Let us consider the particle, with a radius $R$, centered at the origin of the coordinate system, and a fast electron traveling in the proximity of the nanoparticle with velocity $v \sim c$, being $c$ the speed of light in vacuum, and impact parameter $b$, as schematically shown in Fig. 1(a).

The calculation of the total momentum transfer, Eq. (8), requires the knowledge of the total electric and magnetic fields over a closed surface surrounding the particle in order to perform the surface integral. The total electric (magnetic) field outside the particle at any point, is given by superposition of the external electric (magnetic) field, produced by the bare electron, and the induced electric (magnetic) field produced by the induced charges and currents within the particle [see Eq. (10)]. The external electromagnetic fields produced by a bare electron traveling with speed $v$ parallel to the $x$ axis, with $y=0$ and $z=z_{0}=R+b$, are given by ${ }^{27}$

$$
\begin{aligned}
\vec{E}_{e x t}(\vec{r} ; \omega) & \\
= & 2 \frac{-e \omega}{v^{2} \gamma} e^{i \omega x / v}\left\{\frac{1}{\sqrt{y^{2}+\left(z-z_{0}\right)^{2}}} K_{1}\left(\frac{\omega}{v \gamma} \sqrt{y^{2}+\left(z-z_{0}\right)^{2}}\right)\right. \\
& \left.\times\left[y \hat{e}_{y}+\left(z-z_{0}\right) \hat{e}_{z}\right]-\frac{i}{\gamma} K_{0}\left(\frac{\omega}{v \gamma} \sqrt{y^{2}+\left(z-z_{0}\right)^{2}}\right) \hat{e}_{x}\right\},
\end{aligned}
$$




$$
\begin{aligned}
\vec{H}_{e x t}(\vec{r} ; \omega)= & 2 \frac{-e \omega}{v \gamma c} \frac{e^{i \omega x / v}}{\sqrt{y^{2}+\left(z-z_{0}\right)^{2}}} K_{1}\left(\frac{\omega}{v \gamma} \sqrt{y^{2}+\left(z-z_{0}\right)^{2}}\right) \\
& \times\left[-\left(z-z_{0}\right) \hat{e}_{y}+y \hat{e}_{z}\right],
\end{aligned}
$$

where $-e$ is the electric charge of the electron, $\gamma$ $=1 / \sqrt{1-\left(v^{2} / c^{2}\right)}$. Here $K_{0}$ and $K_{1}$ are the modified Bessel functions of order zero and one, respectively. ${ }^{30}$ The electromagnetic induced fields can be obtained by solving Maxwell's equations, using extended Mie theory to obtain analytical expressions for the fields near a single spherical scatterer. The procedure to obtain the expressions consists in expressing the electromagnetic fields in all regions of space in terms of a spherical basis centered at the sphere, and by imposing standard boundary conditions on the surface of the sphere the coefficients of the induced fields can be calculated. An alternative but equivalent formalism based on writing the fields in terms of scalar functions was developed previously. ${ }^{31}$ The explicit expressions of the electromagnetic fields are detailed in the Appendix.

We consider the case of a small particle of radius $R=1 \mathrm{~nm}$ and calculate the differential momentum transfer per unit frequency, $d \vec{P} / d \omega$ [see Eq. (9)], as a function of the frequency. ${ }^{32}$ In this way, we are able to observe directly the main components and sign of the differential momentum transfer that contributes to the total momentum transfer in frequency space, that is, we obtain spectral information of the particle response to the broadband probing electron. The validity of the dielectric formalism for such small particles has been proven to be adequate in similar nanoparticle sizes (silver particles of size down to 1-2 nm) with extremely good agreement between the energy of the plasmon losses theoretically calculated within the dielectric formalism and the energy loss peaks experimentally obtained in electronenergy-loss spectroscopy. ${ }^{2,9,33,34}$ The total momentum transfer is a vector but due to the symmetry of the problem there is no momentum transfer in the $y$ component [see Fig. 1(a)]. In Fig. 2(a) we show $d P_{x} / d \omega$ as a function of the frequency, that is, the $x$ component (the longitudinal component along the electron trajectory) of the differential momentum transfer per unit frequency, for several impact parameters between $b=0.5$ and $10 \mathrm{~nm}$. We observe that the dipolar plasmon mode $(l=1)$, located at $\hbar \omega \approx \hbar \omega_{p} / \sqrt{3} \approx 8.72 \mathrm{eV}$, is the most efficiently excited mode, but a quadrupolar, $l=2$, $\quad(\hbar \omega$ $\approx 9.55 \mathrm{eV})$ and octopolar, $l=3$, $(\hbar \omega \approx 9.89 \mathrm{eV})$ plasmon modes can also be distinguished, particularly for the closest impact parameter $b=0.5 \mathrm{~nm}$. In the inset of Fig. 2(a) we show the total momentum transfer in the $x$ direction as a function of the impact parameter $b$, obtained after integration for each impact parameter $b$ [see Eq. (8)]. For large values of $b$, the electromagnetic fields produced by the fast electron excite poorly the surface plasmon modes of the sphere, and there is practically no momentum transfer to the particle. However, as the electron passes closer to the particle, the momentum transfer increases monotonically and quickly, particularly for impact parameters $b \leqq 5 \mathrm{~nm}$.

The differential momentum transfer per unit frequency along the $z$ component, $d P_{z} / d \omega$, which corresponds to the direction perpendicular to the electron trajectory, is shown in
Fig. 2(b) as a function of frequency for the same range of values of the impact parameter as in Fig. 2(a). We observe that the spectral structure of $d P_{z} / d \omega$ corresponds to a superposition of several resonant modes, each one having a Lorentzian derivative shape [see Fig. 2(b)]. Each resonant mode corresponds to a plasmon mode, thus in Fig. 2(b) we can clearly distinguish the excitation of three lower plasmon modes [indicated in Fig. 2(b) by the first three black arrows, from left to right]. For each plasmon mode $l$ the contribution to the momentum transfer of frequencies lower than the resonance frequency $\omega_{l}$ is positive or, in other words, these contributions to the momentum transfer induce an attractive force between the electron and the particle. For frequencies larger than $\omega_{l}$ the momentum transfer produces a repulsive force. Here by the term attractive we want to stress that the particle will move toward the electron trajectory (positive direction of $z$ axis), while by the term repulsive we denote that the particle will move away from the electron trajectory (negative direction of $z$ axis). This response is similar to the dispersive coupling in a harmonic oscillator with $\omega<\omega_{l}$ attractive and $\omega>\omega_{l}$ repulsive. The line shape of the momentum transfer spectrum corresponding to the excitation of the plasmon modes can be understood by looking at the analytical expression of the transverse force in the case of a nonretarded electromagnetic interaction, ${ }^{35}$ which depends only on the real part of the $\alpha_{l}$ polarizability,

$$
F_{\perp} \sim \operatorname{Re}\left[\alpha_{l}(\omega)\right]=\operatorname{Re}\left\{\frac{[1-\varepsilon(\omega)] l}{l \varepsilon(\omega)+l+1}\right\} .
$$

Substituting the Drude dielectric function, Eq. (11), into Eq. (14) and plotting it as a function of the frequency, exhibits the same Lorentzian derivative shape around the modes, as the ones shown in Fig. 2(b). Even if Eq. (14) is a quasistatic approach of the transverse force that serves to understand in simple terms the spectral line shape around the resonances, it is important to stress that all the calculations presented in this work are performed by fully solving Maxwell's equations, therefore, in a full electrodynamical treatment including retardation effects. Integrating over the frequency the contribution of all plasmon modes (which has to be done very carefully due the fine cancellations between positive and negative contributions), we obtain the total momentum transfer for each impact parameter $b$. In this way, it is possible to know if the total interaction between the electron and the particle will be attractive or repulsive.

We show the $z$ component of the total momentum transfer as a function of the impact parameter $b$, shown as an inset in Fig. 2(b), where we observe that for large impact parameters there is practically no momentum transfer. The total momentum transfer increases slowly as the electron trajectory approaches to the particle, up to a maximum located around $b \approx 1 \mathrm{~nm}$. For smaller impact parameters the momentum transfer decreases abruptly, changing sign and becoming repulsive. In Figs. 2(a) and 2(b), we can observe that $d P_{x} / d \omega$ (longitudinal component) tends to zero in the limit $\omega \rightarrow 0$, while $d P_{z} / d \omega$ (transverse component) tends to a constant value, in a good agreement with previous reports on the longitudinal force experienced by fast electrons near interfaces ${ }^{26,35}$ and small particles. ${ }^{16}$ Both the multipolar na- 

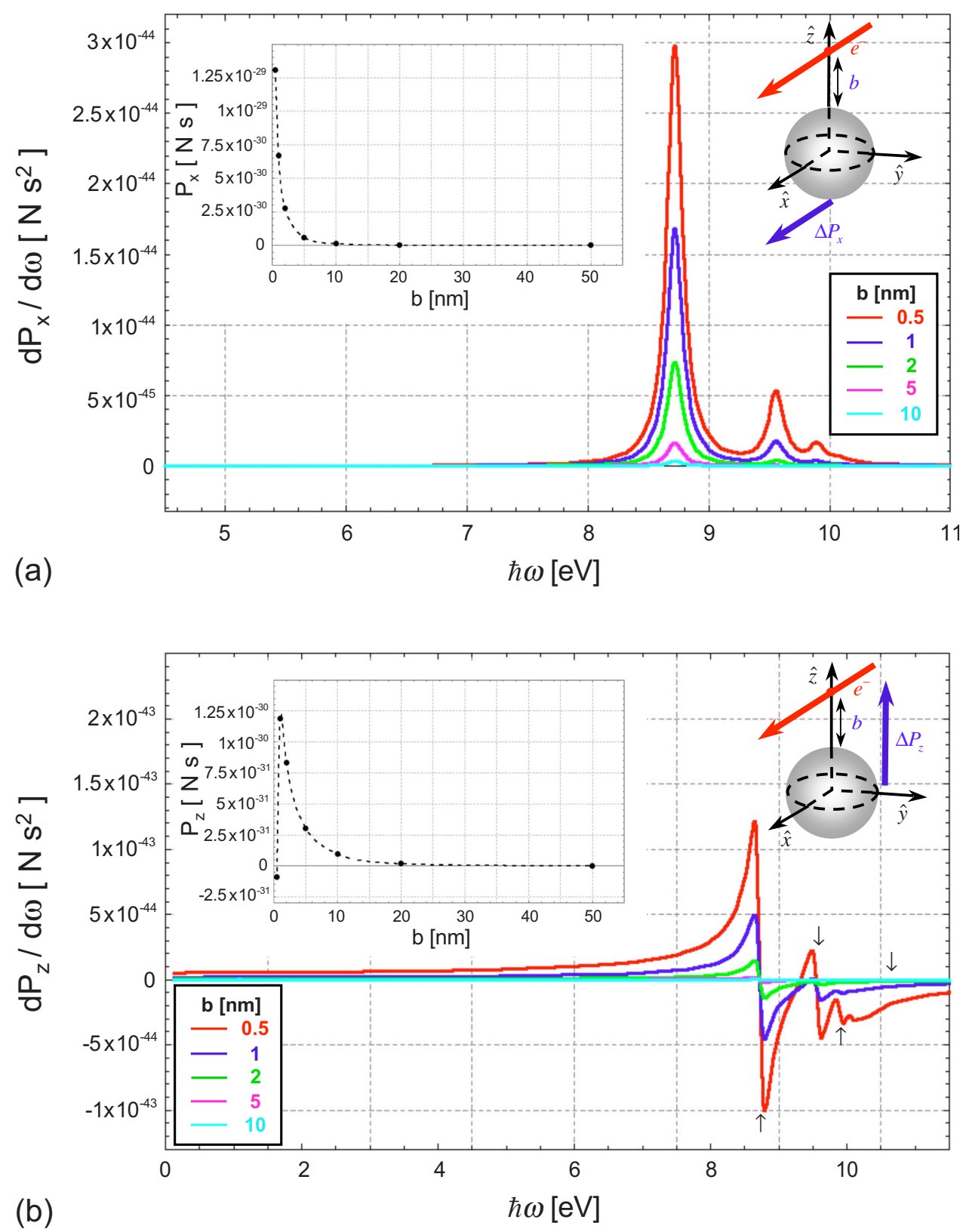

FIG. 2. (Color online) Frequency contribution to (a) $x$ and (b) $z$ components of the momentum transfer from the fast electron to a single Drude-type spherical particle of radius $R=1 \mathrm{~nm}$, for different impact parameters $b$. Inset: (a) $x$ and (b) $z$ components of the total momentum transfer as a function of the impact parameter $b$. The dashed line is a guide to the eyes. ture of the excitations at small impact parameter and the full retarded description of the interaction are involved in the result of a repulsive force (negative sign). Variations in the swift electron velocity $v$ within the range of the typical energies used in STEM experiments do change the magnitude of the momentum transfer (a few times larger for smaller velocities) but do not modify the main features of attraction and repulsion of the forces induced. However, for very low electron velocities, spectral changes in the momentum transfer can be observed with positive net force even for small impact parameters. In this case, the effect of retardation in the multipolar response is reduced.

To understand and corroborate the nature of each plasmon mode in Figs. 2(a) and 2(b), we display the magnitude of the induced electric near field around the spherical particle, for the frequencies of the modes indicated by arrows in Fig. 2(b). We select an impact parameter $b=0.5 \mathrm{~nm}$ as representative of a very-close trajectory. The four contour plots of the near field, shown in Fig. 3, correspond to the induced electric field in the $x=0$ plane, i.e., in the $Y Z$ plane perpendicular to the electron trajectory (indicated by a black arrow in each of the four near-field plots in Fig. 3) and intersecting the center of the nanoparticle. In addition to the contour plot, a projection of the field in the $Y Z$ plane is displayed on top of each graph for a better appreciation of the distribution of the induced electric field around the particle. The top-left plot in Fig. 3 corresponds to a frequency of $\hbar \omega=8.72 \mathrm{eV}$, where the lower energy plasmon mode is located [see Fig. 2(b)]. The spatial distribution of the near field confirms that this mode is indeed a dipolar plasmon mode. This is in a good agreement with the theoretical prediction of the $l$ modes for a small spherical particle, located at frequencies $\omega_{l}$ $=\omega_{p} \sqrt{l /(2 l+1)}$, where $l=1$ corresponds to the dipolar plasmon mode. The quadrupolar plasmon mode is located at $\hbar \omega=9.55 \mathrm{eV}$ when $l=2$, consistent with the position of the mode in Fig. 2(b), and also consistent with the induced field 



FIG. 3. (Color online) Threedimensional (3D) plot and 2D projection on top, of the magnitude of the induced electric field in the vicinity of a Drude-type particle of radius $R=1 \mathrm{~nm}$, with an impact parameter of the electron trajectory (indicated by a black arrow) of $b=0.5 \mathrm{~nm}$, for four selected values of the frequency denoted by arrows in Fig. 2(b). The electric field is expressed in atomic units.

pattern shown in the top right of Fig. 3 with four clear peaks around the particle. At a frequency of $\hbar \omega=9.89 \mathrm{eV}$, corresponding to $l=3$, the identification of the octopolar plasmon mode in the near-field plot (see bottom-left plot in Fig. 3) is not so straightforward, due to the superposition of higher multipolar plasmon modes that lie very close in energy. The planar surface plasmon mode $\omega_{s}=\omega_{p} / \sqrt{2}$ (which corresponds to the limit $l \rightarrow \infty$ ) is located at $\hbar \omega=10.68 \mathrm{eV}$ in aluminum. It is possible to observe in the bottom-right plot in Fig. 3 how the induced electric field is concentrated in a small region closer to the electron trajectory, consistent with the high-order multipolar nature of this peak.

To evaluate the strength of the forces for different particle sizes, we also study the case of a larger spherical particle of radius $R=40 \mathrm{~nm}$. We also characterized the particle by a Drude-type dielectric function with the same parameters $\omega_{p}$ $=15.1 \mathrm{eV}$ and $\Gamma=0.15 \mathrm{eV}$. The particle is centered at the origin of the coordinate system and surrounded by vacuum. We assume a fast electron traveling with the same velocity $v$. The differential momentum transfer per unit frequency along the $x$ component, $d P_{x} / d \omega$ (longitudinal component), for the $R=40 \mathrm{~nm}$ Drude-type spherical particle, is shown in Fig. 4(a). Compared with the small particle case, it can be observed that more plasmon modes are activated and that the dipolar plasmon mode is not longer the most efficiently excited mode. ${ }^{15}$ In this case, the higher order (larger energy) plasmon modes have an important contribution to the momentum transfer. One can also see in Fig. 4(a) that, while the lower plasmon modes give contribution along the particle direction to the total momentum transfer (positive sign in the spectrum), higher multipolar modes give a contribution in the contrary direction (negative sign in the spectrum). Integrating to all frequencies we obtain the total momentum transfer for each impact parameter $b$, shown in the inset of Fig. 4(a). For large impact parameters there is practically no momentum transfer, but as the electron gets closer to the particle, the momentum transfer increases quickly, in a similar way as in the small particle case [see Fig. 2(a)]. However, in the case of a big particle, we observe that the momentum transfer along the particle trajectory reaches a maximum located at around $b \approx 2 \mathrm{~nm}$ and then decreases rapidly for closer impact parameters. The momentum transfer along the $x$ component remains always positive, or in terms of the interaction, the particle is always pushed along the electron trajectory. Compared with the small particle case, the momentum transfer in this case $(R=40 \mathrm{~nm})$ is around one order of magnitude larger.

The $z$ component (transverse to the electron trajectory) of the differential momentum transfer per unit frequency, $d P_{z} / d \omega$, for the big particle case $(R=40 \mathrm{~nm})$ is shown in Fig. 4(b). As for the $x$ component [see Fig. 4(a)], the higher order plasmon modes are excited very efficiently, and similarly, the lower energy modes give an attractive contribution to the total momentum transfer while the higher energy modes give a repulsive contribution. This behavior can be understood as a superposition of a Lorentzian derivativelike shape for each plasmon mode, overlapping the negative region for frequencies larger than the frequency $\omega_{l}$ of each mode $l$, with the positive region of the subsequent mode, for frequencies below $\omega_{l+1}$. The plasmon modes are located closer to each other as $l$ increases, therefore when these higher energy plasmon modes are excited, i.e., when there is large density of states of these higher energy modes, the negative region overcomes the value of the positive region of the next mode. Even though more plasmon modes are activated, the total momentum transfer as a function of the impact parameter [see inset of Fig. 4(b)] shows a similar behavior (although smoother) as in the small particle case [see inset of Fig. 2(b)], i.e., for large impact parameters there is practically no momentum transfer, and it increases slowly as the electron trajectory gets closer to the particle. A maximum for the momentum transfer is reached (at around $b \approx 6 \mathrm{~nm}$ for the big particle case), then the momentum transfer de- 
(a)

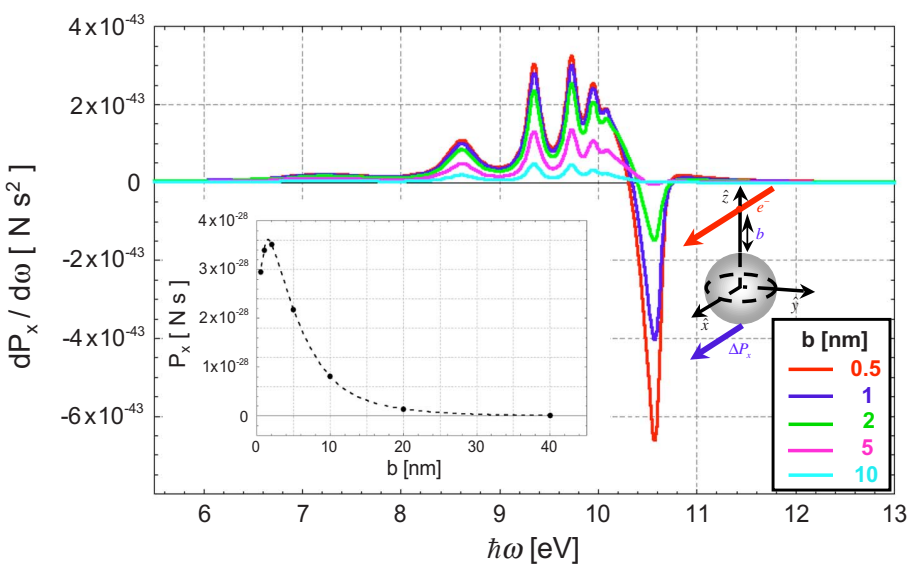

(b)



(c)
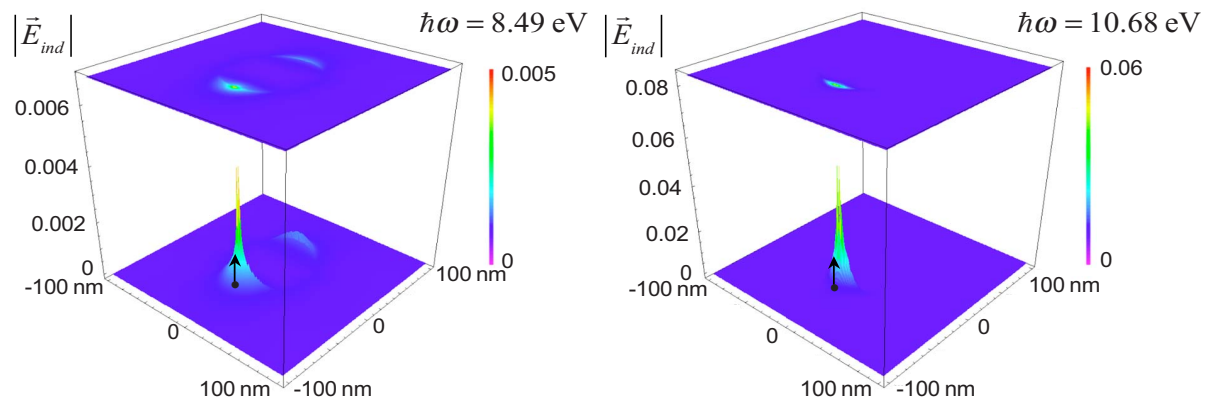

FIG. 4. (Color online) Frequency contribution to (a) $x$ and (b) $z$ components of the momentum transfer from the fast electron to a single Drude-type spherical particle of radius $R=40 \mathrm{~nm}$, for different impact parameters $b$. Inset: (a) $x$ and (b) $z$ components of the total momentum transfer as a function of the impact parameter $b$. The dashed line is a guide to the eyes. (c) 3D plot and 2D projection on top, of the magnitude of the induced electric field in the vicinity of a Drude-type particle of radius $R=40 \mathrm{~nm}$, with an impact parameter of the electron trajectory (indicated by a black arrow) of $b=0.5 \mathrm{~nm}$, for two selected values of the frequency denoted by arrows in (b). creases rapidly for smaller impact parameters, becoming repulsive for very small impact parameters $(b \approx 3 \mathrm{~nm})$. Numerically, the momentum transfer in the $z$ component for this case $(R=40 \mathrm{~nm})$ is around three orders of magnitude larger than the small particle case. We also calculate the induced electric near field around the particle $(R=40 \mathrm{~nm})$, for the two frequencies indicated by an arrow in Fig. 4(b) (impact parameter $b=0.5 \mathrm{~nm})$. The near-field plots shown in Fig. 4(c), correspond to the induced electric field in the $Y Z$ plane perpendicular to the electron trajectory [indicated by a black arrow in each of the two near-field plots in Fig. 4(c)]. The two-dimensional (2D) projection of the induced near field in the $Y Z$ plane is displayed on the top of their respective contour plots. From the near-field spatial distribution of the lowest-energy (dipolar) plasmon mode at $\hbar \omega=8.49 \mathrm{eV}$ [see left-hand side in Fig. 4(c)], it is difficult to identify the nature of the plasmon mode. The reason for this is that the electric field cannot excite efficiently the dipolar mode that also in- volves the excitation of the farthest side of the particle, opposite to the electron trajectory. The plasmon modes are redshifted [see Fig. 4(b)] for large particles with respect to those in the small particles [see Fig. 2(b)] due to the effect of retardation. At the energy of the planar surface plasmon mode, $\hbar \omega=10.68 \mathrm{eV}$, it is possible to observe a strong localization of the induced electric field in the region nearby the electron trajectory [see right-hand side in Fig. 4(c)], similar to the small particle case (bottom-right plot in Fig. 3). The strength of the induced field for this higher order mode is about one order of magnitude larger than the strength of the induced field associated with the dipolar mode. The values for both $d P_{x} / d \omega \rightarrow 0$ and $d P_{z} / d \omega \rightarrow$ constant in the limit of $\omega \rightarrow 0$ can also be observed in this case. Interestingly, the longitudinal component of the momentum transfer also presents a negative contribution at the position of the planar surface plasmon $\left(\omega_{s} \approx 10.68 \mathrm{eV}\right)$, similarly to the transverse component. This is an effect of the higher order multipoles. 


\section{B. Gold nanoparticle}

To apply the concept of attractive and repulsive range of impact parameters in a realistic situation, let us consider a single small gold nanoparticle of radius $R=1 \mathrm{~nm}$. We calculate the differential momentum transfer per unit frequency for both $x$ (longitudinal) and $z$ (transverse) directions for different impact parameters between $b=0.3 \mathrm{~nm}$ and $b=2 \mathrm{~nm}$, as shown in Figs. 5(a) and 5(b), respectively. We use the bulk experimental data ${ }^{29}$ for the dielectric response of gold. This response includes damping and has been shown to be good for particles down to $1 \mathrm{~nm}$. Below $1 \mathrm{~nm}$, a more complex behavior that involves nonlocal effects and size-dependent damping can be expected but this goes beyond the scope of the present work. Both components of the momentum transfer, $x$ and $z$, have a very different frequency behavior compared to the Drude-type particle case due to the more complex spectral response of gold driven by interband transitions. Here the plasmon modes are not so clearly identified and, for example, in the transverse component of the momentum transfer, the Lorentzian derivativelike shape displayed in Drude-type particle case is lost. The contribution to the total momentum transfer of the low-frequency modes, in this case is not the most relevant. The momentum transfer along the direction of the electron trajectory ( $x$ component) is dominated by excitations of energy between 10 and $60 \mathrm{eV}$, while in the case of the $z$ component, we can observe that the low-energy modes, less than $25 \mathrm{eV}$, gives an attractive contribution to the total momentum transfer, and for larger frequencies the contribution is dominantly repulsive. We also observe that, for both $x$ and $z$ components, it is necessary to integrate up to very high energies in the frequency domain to obtain the total momentum transfer. Nevertheless, the total momentum transfer as a function of the impact parameter $b$ for both $x$ and $z$ components, behaves in a similar way to the small Drude-type particle case, with values of around one order of magnitude larger for the gold case. For the $x$ component (longitudinal), the total momentum transfer as a function of the impact parameter $b$ [shown as an inset in Fig. $5(\mathrm{a})$ ], increases monotonically as the electron gets closer to the particle, while for the $z$ component, shown as an inset in Fig. 5(b), there is a maximum located around $b \approx 1 \mathrm{~nm}$ [as in the small Drude-type spherical particle, see Fig. 2(b)] and then a rapid decrease for smaller impact parameters, changing sign and becoming repulsive as the electron trajectory gets closer to the particle. In Fig. 5(c) we show two nearfield plots for the induced electric field, for selected frequencies as indicated by arrows in Fig. 5(b). For the low-energy plasmon mode, $\hbar \omega=2.3 \mathrm{eV}$, it is not possible to identify the nature of the plasmon mode since several surface modes pile up around this energy value. For larger energy $\hbar \omega=38 \mathrm{eV}$, we observe a strong localization of the field in the region closer to the electron trajectory. Finally, as a general trend, we can conclude that the momentum transfer for metallic particles with large electron impact parameters pulls the particle toward the electron beam, whereas for closer impact parameters, where higher order multipole modes are excited, the particle is pushed away from the electron beam.

\section{MOMENTUM TRANSFER IN A NANOPARTICLE DIMER}

We analyze now the effects of the presence of a second particle in the momentum transfer. In the case of an electron beam passing near a metallic dimer, we consider the geometry depicted in Fig. 1(b), consisting of two identical spherical particles characterized by a Drude-type dielectric function. We study two cases: a dimer of two small particles and a dimer of two big particles. As a realistic situation, we also consider a gold dimer. We calculate the momentum transfer to the top particle of the dimer (closest one to the electron beam), following a similar strategy as in the one singleparticle case. The fast electron produces the external field (expressions introduced in the previous section) that induces surface charges and currents at the surface of the particles. We focus on the top particle since the momentum transferred to the bottom particle (furthest one with respect to the electron beam) is much smaller in magnitude due to the larger distance to the electron beam. The coalescence effect therefore will be governed basically by the force on the top particle. Due to the complexity of the dimer geometry, we calculate numerically the induced fields around the particles with use of the boundary element method. ${ }^{36,37}$ We explore the momentum transfer as a function of the impact parameter $b$ and also as a function of the separation distance between the particles in the dimer, $d$. We also analyzed the effect of the size of particles in this dimer configuration.

\section{A. Drude-type nanoparticle dimer}

We study first the case of two identical small particles in a dimer configuration, as shown in Fig. 1(b). The radii are $R=1 \mathrm{~nm}$ and the particles are separated by a distance $d$ $=0.25 \mathrm{~nm}$. We consider the dimer embedded in vacuum and both particles characterized by the Drude response [see Eq. (11)] with $\omega_{p}=15.1 \mathrm{eV}$ and $\Gamma=0.15 \mathrm{eV}$. We calculate numerically the electromagnetic fields self-consistently, i.e., considering the interactions between the electron and the two particles in the dimer, and calculate the momentum transfer from the electron to the top particle in the dimer [see Fig. 1(b)]. The results of $d P_{x} / d \omega$ (longitudinal component) and $d P_{z} / d \omega$ (transverse component) as a function of $\omega$, for several impact parameters in the range $b=0.5-10 \mathrm{~nm}$, are shown in Figs. 6(a) and 6(b), respectively. The momentum transfer is calculated for the top particle, highlighted with a solid thick circle in the insets of both Figs. 6(a) and 6(b).

For $d P_{x} / d \omega$ as a function of $\omega$ [see Fig. 6(a)], we observe a similar behavior as in the case of a single small Drude sphere [see Fig. 2(a)], but a more complex modal structure and a redshift of the positions of the resonances is present due to hybridization of the modes of the two spheres. For example, the lowest-energy resonance for the single-particle case is located around $\hbar \omega=8.72 \mathrm{eV}$, while for the dimer it is located around $\hbar \omega=7.4 \mathrm{eV}$, which is a hybridized bonding plasmon localized at the junction between the two spheres. ${ }^{17,20,21}$ The momentum transfer to the top particle in the dimer, obtained after integration over all frequencies, is plotted as a function of the impact parameter $b$, and shown as an inset in Fig. 6(a), exhibiting a similar behavior as for the 
(a)

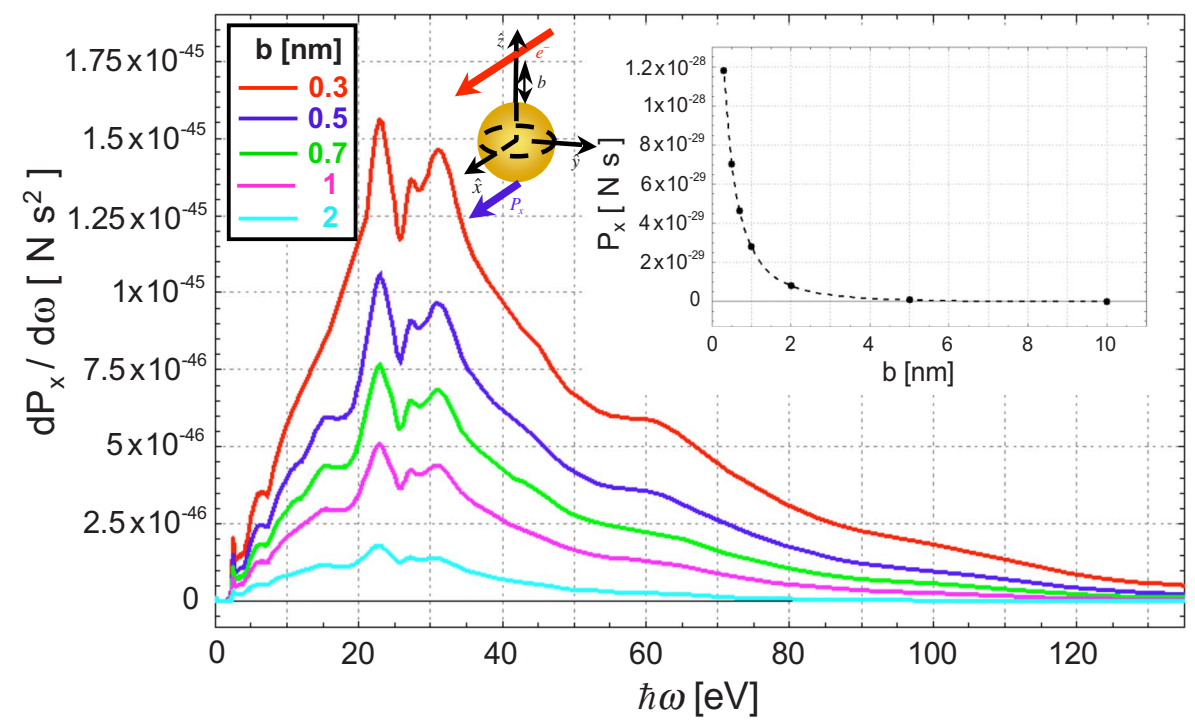

(b)



(c)
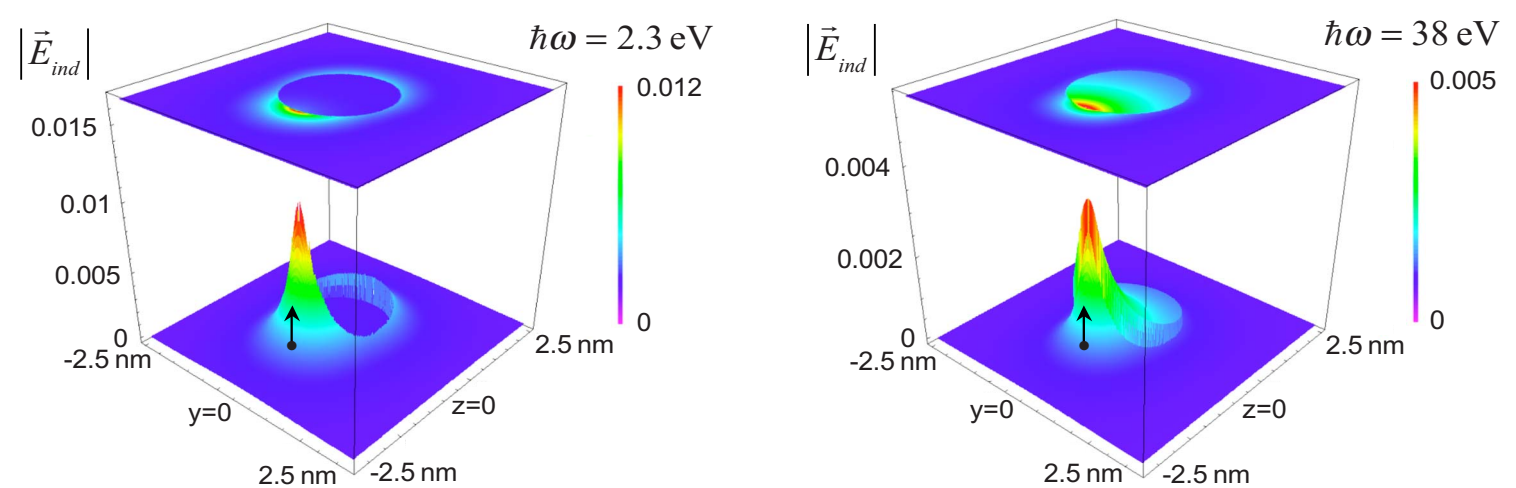

FIG. 5. (Color online) Frequency contribution to (a) $x$ and (b) $z$ components of the momentum transfer from the fast electron to a single gold spherical particle of radius $R=1 \mathrm{~nm}$, for different impact parameters $b$. Inset: (a) $x$ and (b) $z$ components of the total momentum transfer as a function of the impact parameter $b$. The dashed line is a guide to the eyes. (c) 3D plot and 2D projection on top, of the magnitude of the induced electric field in the vicinity of a gold particle of radius $R=1 \mathrm{~nm}$, with an impact parameter of the electron trajectory (indicated by a black arrow) of $b=0.3 \mathrm{~nm}$, for two selected values of the frequency denoted by arrows in (b). 
(a)

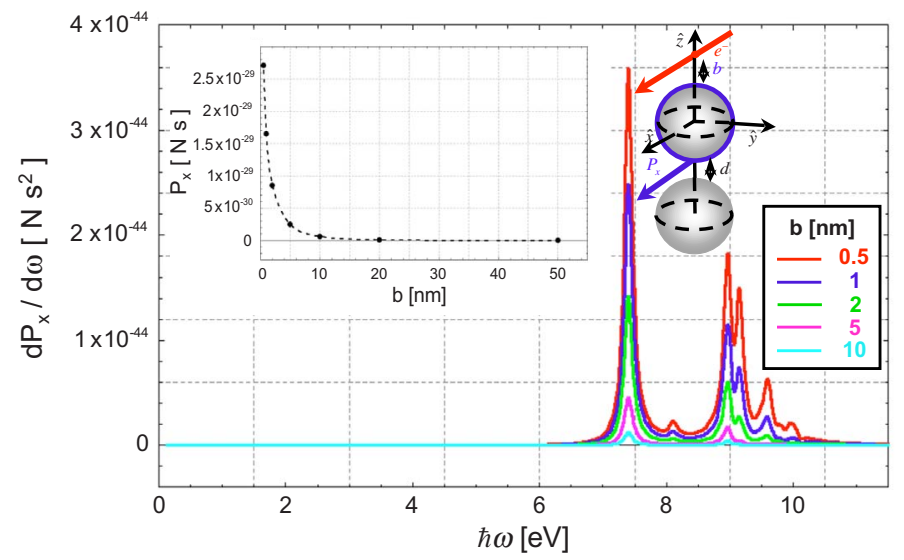

(b)

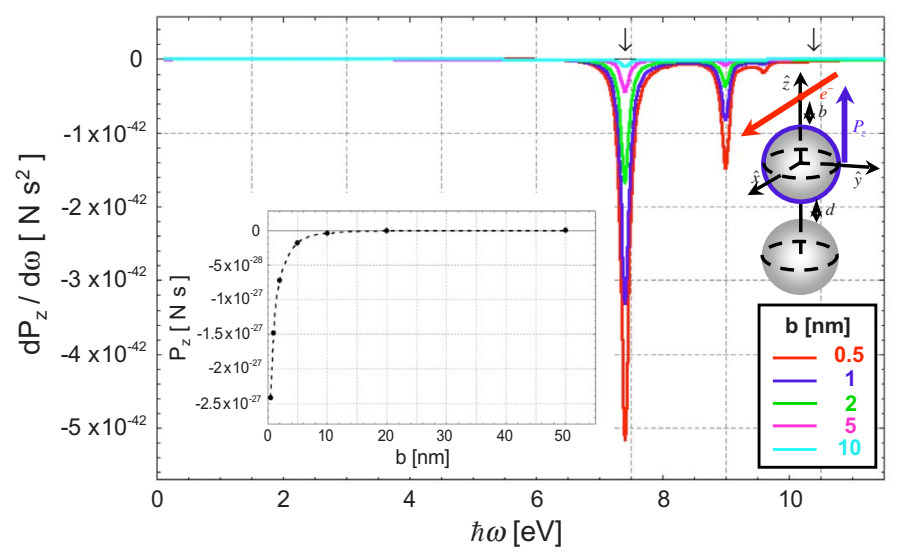

(c)
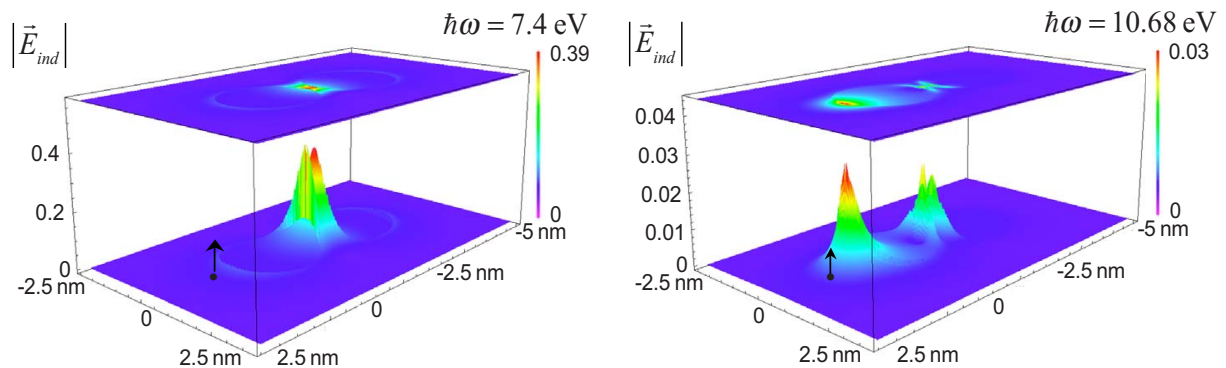

FIG. 6. (Color online) Frequency contribution to (a) $x$ and (b) $z$ components of the momentum transfer from the fast electron to a top Drude-type spherical particle in a dimer configuration of identical particles of radius $R=1 \mathrm{~nm}$ separated by a distance $d=0.25 \mathrm{~nm}$, for different impact parameters $b$. Inset: (a) $x$ and (b) $z$ components of the momentum transfer to the top particle in the dimer as a function of the impact parameter $b$. The dashed line is a guide to the eyes. (c) 3D plot and 2D projection on top, of the magnitude of the induced electric field in the vicinity of a dimer of identical Drude-type particles of radius $R=1 \mathrm{~nm}$, separated by a distance $d=0.25 \mathrm{~nm}$ with an impact parameter of the electron trajectory (indicated by a black arrow) of $b=0.3 \mathrm{~nm}$, for two selected values of the frequency denoted by arrows in (b).

one small single-particle case, i.e., a monotonic decrease in momentum transfer as the electron gets further from the dimer. However, the spectra for the transverse component, along the direction of the dimer axis, $d P_{z} / d \omega$ [see Fig. 6(b)] presents a totally different trend, compared to the case of a small single particle. In this case, the resonances, though redshifted, do not exhibit a Lorentzian derivativelike shape and the contribution to the momentum transfer is repulsive at practically all frequencies. This functional dependence has an impact in the momentum transferred to the top particle, as a function of the impact parameter [see inset in Fig. 6(b)], where we observe that the interaction between the electron and the top particle is repulsive for all impact parameters considered. It is clear that in this case, the generation of the bonding dimer modes associated with the dimer interaction governs the physics of the momentum transfer. We can analyze deeper the nature of the modes excited in this case. In Fig. 6(c), we show two near-field plots of the magnitude of the induce electric field around the two spheres, for selected frequencies marked by the arrows in the spectrum of Fig. 


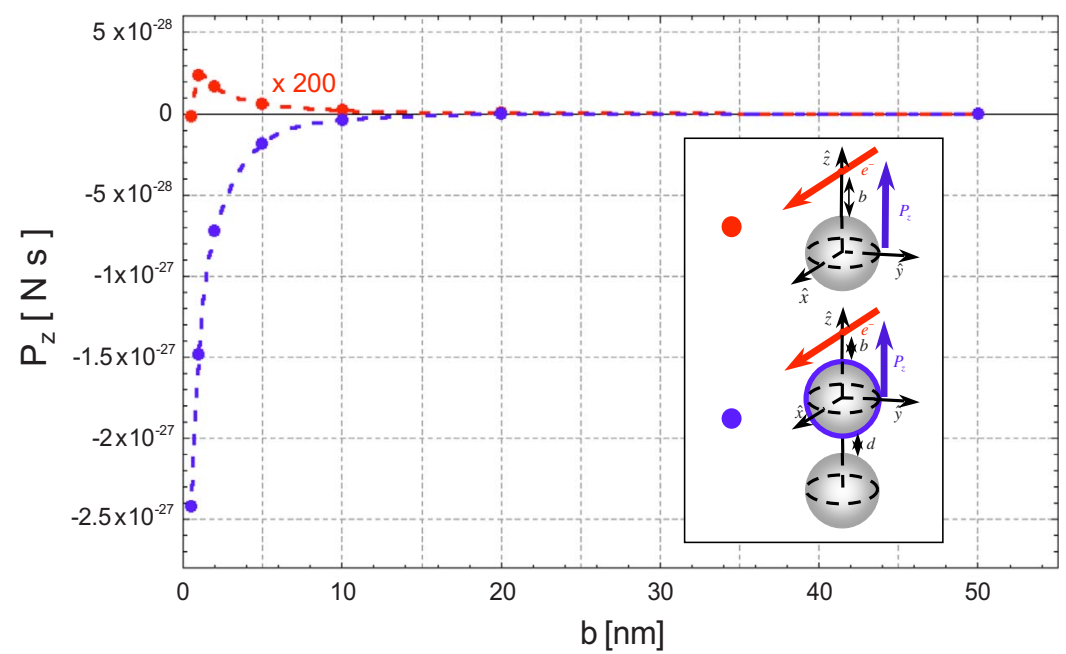

FIG. 7. (Color online) Comparison of total momentum transfer along $z$ direction as a function of the impact parameter $b$, between a single small $(R=1 \mathrm{~nm})$ Drude-type particle (multiplied by a factor of 200 for a better appreciation) and the top particle in a dimer of small $(R=1 \mathrm{~nm})$ Drude-type identical particles separated by a distance $d=0.25 \mathrm{~nm}$. Dashed lines are a guide to the eyes. 6(b). For the plasmon mode located at $\hbar \omega=7.4 \mathrm{eV}$, the nearfield plot [see left plot in Fig. 6(c)] shows a strong interaction between the two particles, that is, a strong enhancement of the induced electric field in the space between the two particles. This is a fingerprint of a bonding dimer plasmon that is very effectively excited as a consequence of the proximity of the two spheres, piling up charge density at the dimers cavity. For higher frequencies, close to the planar surface plasmon $\hbar \omega=10.68 \mathrm{eV}$, the field is concentrated in the region close to the electron trajectory due to the delocalization of these higher energy modes.

Since the main features in a dimer are determined by the bonding plasmons formed at the cavity, one can expect a smaller dependence of the sign of the force as a function of impact parameter. In Fig. 7, we compare the total momentum transfer in the direction transverse to the electron trajectory for the single-particle case and the top particle in the dimer (radii $R=1 \mathrm{~nm}$ ) as a function of electron impact parameter. We can clearly see the effect of locating a second particle below: in the case of one single particle, the interaction between the electron and the particle is repulsive only for extremely close electron trajectories. When placing a second particle below, the interaction between the top particle and the electron becomes repulsive for all the impact parameters considered. The effect of the presence of a second particle below is to pull the top particle down, toward the bottom particle, thanks to the excitation of bonding dimer plasmons localized in the cavity between both particles. This effect can be qualitatively understood by considering this induced charge associated with the bonding mode at the gap, which present opposite charges on adjacent spheres. Furthermore, in the quantitative analysis of the effect, it is remarkable that for the closest impact parameter $b=0.5 \mathrm{~nm}$, the momentum transfer in the dimer case due to the presence of the bottom particle is four orders of magnitude more repulsive than for the case of a single particle.

To activate higher order modes within the cavity, we also consider the case of larger identical particles in a dimer configuration, with radius $R=40 \mathrm{~nm}$ and separation distance $d=1 \mathrm{~nm}$. We calculate the differential momentum transfer (to the top particle in the dimer) per unit frequency along both directions $d P_{x} / d \omega$ and $d P_{z} / d \omega$, shown in Figs. 8(a) and 8(b), respectively. For the $x$ component [in Fig. 8(a)] similarly to the small dimer, a more complicated structure of the modes (modes pile up) can be observed when compared with the case of a big single particle [see Fig. 4(a)], alternating the sign between the contribution of each plasmonic mode. Due to the size of the particles, the lower energy hybridized modes in the system are not efficiently excited, thus in this case the redshift of the modes is harder to observe. Integrating the contribution to all frequencies, we obtain the momentum transfer $P_{x}$ to the top particle in the dimer as a function of the impact parameter $b$ [inset of Fig. 8(a)]. showing a similar behavior as for the single-particle case. This means that the high-order modes responsible for the longitudinal force in large particles are very similar both for single particles and particles in dimers due to their delocalization. Moreover, quantitatively the momentum transfer for both cases: single particle and top particle in the dimer [see Figs. 4(a) and 8(a)], is of the same order of magnitude, and we can see that in both cases the maximum of intensity of the momentum transfer as a function of the impact parameter is reached at around $b=2 \mathrm{~nm}$. This implies that the presence of the second particle is not relevant for the longitudinal momentum transfer in big particles. For the $z$ component [see Fig. 8(b)], we also observe a more complicated dependence on frequency of $d P_{z} / d \omega$, in comparison with the singleparticle case, and the redshift of the modes is also hard to observe in the spectrum. However, the high-energy modes still contribute with negative sign (attractive force between particles) to the momentum transfer. The momentum transfer $P_{z}$ to the top particle in the dimer as a function of the impact parameter $b$, inset in Fig. 8(b), shows relevant differences between the dimer and single-particle cases: in the case of the dimer, the momentum transfer results in a repulsive force between top particle and electron (attractive force between particles) for all impact parameters considered while in the single-particle case the force between particle and electron becomes repulsive only for impact parameters smaller than $3.5 \mathrm{~nm}$. We also notice that there is no significant difference in magnitude between the total momentum transfer of one single-particle and dimer cases, in contrast with the small particle calculations, meaning that there is an overall smaller influence of the bonding localized plasmons in large par- 
(a)

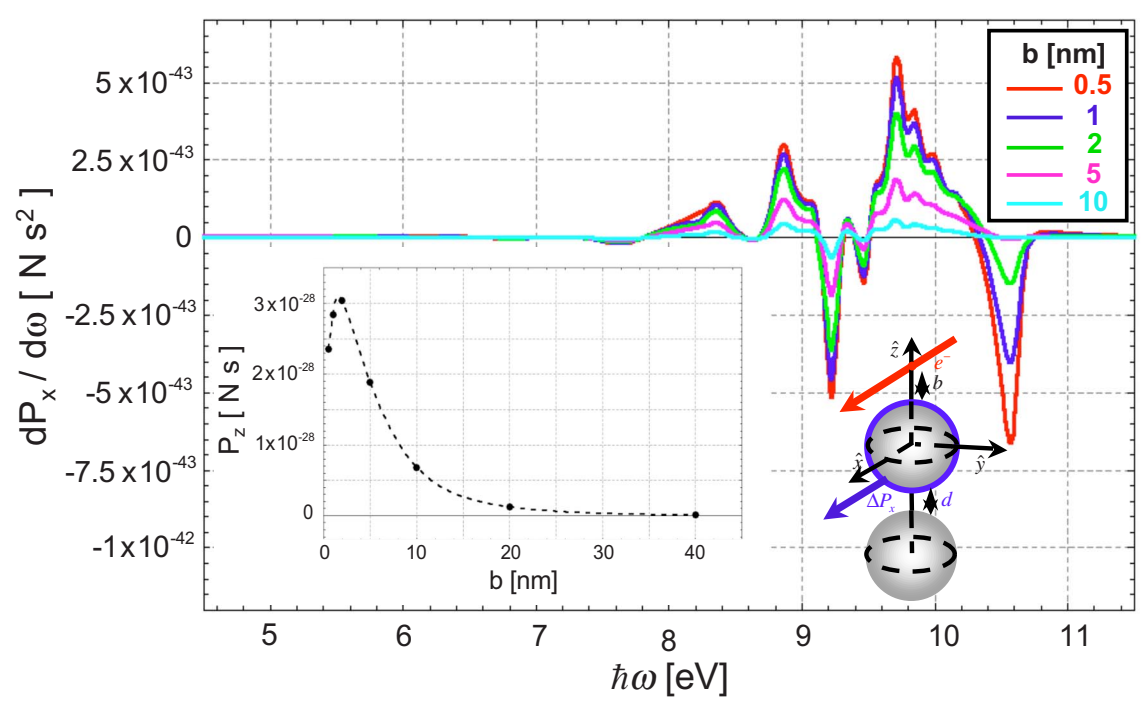

(b)

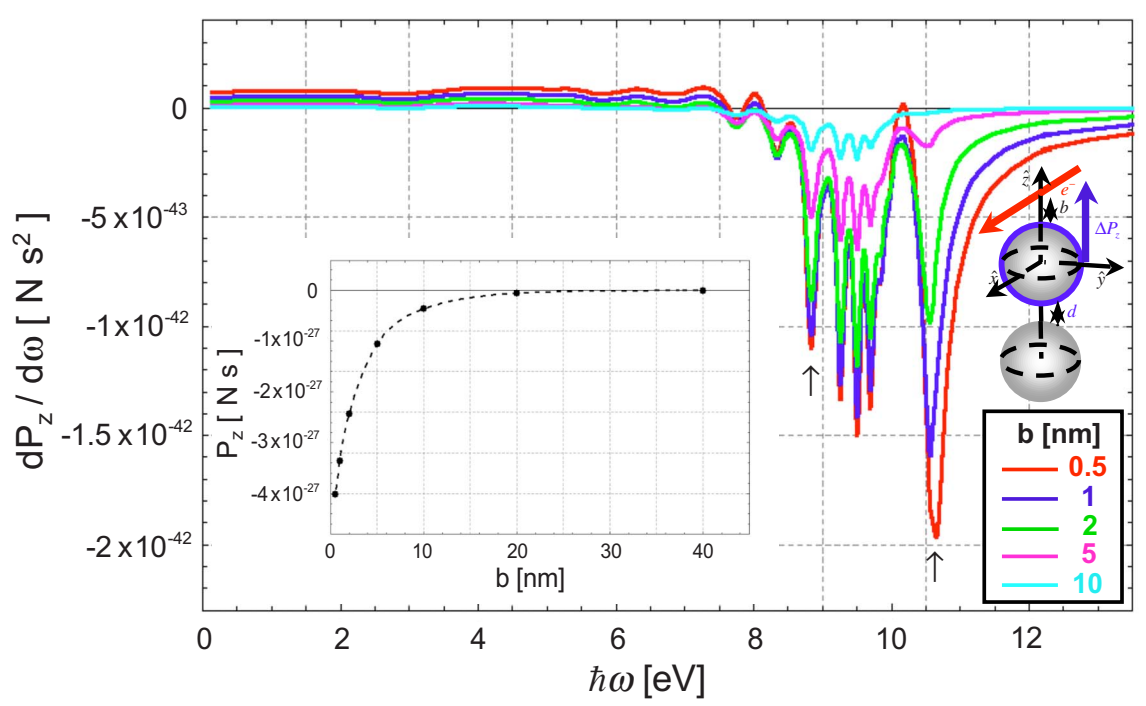

(c)


FIG. 8. (Color online) Frequency contribution to (a) $x$ and (b) $z$ components of the momentum transfer from the fast electron to a top Drude-type spherical particle in a dimer configuration of identical particles of radius $R=40 \mathrm{~nm}$ separated by a distance $d=1 \mathrm{~nm}$, for different impact parameters $b$. Inset: (a) $x$ and (b) $z$ components of the momentum transfer to the top particle in the dimer as a function of the impact parameter $b$. The dashed line is a guide to the eyes. (c) 3D plot and 2D projection on top, of the magnitude of the induced electric field in the vicinity of a dimer of identical Drude-type particles of radius $R=40 \mathrm{~nm}$, separated by a distance $d=1 \mathrm{~nm}$ with an impact parameter of the electron trajectory (indicated by a black arrow) of $b=0.5 \mathrm{~nm}$, for two selected values of the frequency denoted by arrows in (b). 


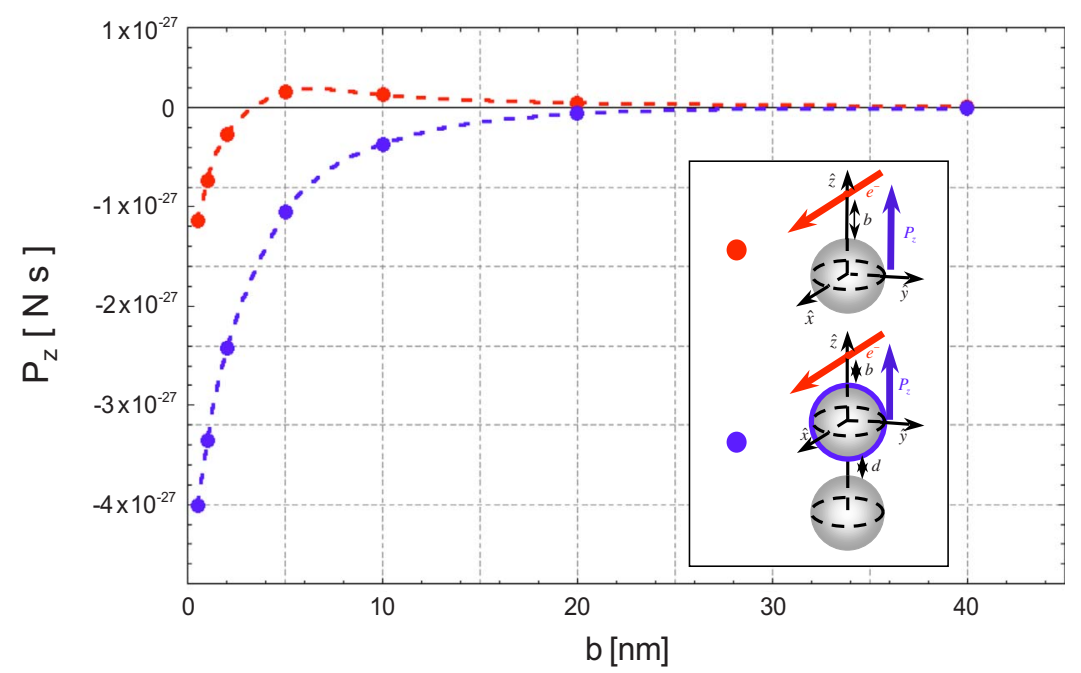

FIG. 9. (Color online) Comparison of total momentum transfer along $z$ direction as a function of the impact parameter $b$, between a single large $(R=40 \mathrm{~nm})$ Drude-type particle and the top particle in a dimer of large $(R=40 \mathrm{~nm})$ Drudetype identical particles separated by a distance $d$ $=1 \mathrm{~nm}$. Dashed lines are a guide to the eyes.

ticles, as expected. In Fig. 8(c) we plot the magnitude of the induced field around the particles for two frequencies marked by arrows in Fig. 8(b). For low frequency $\hbar \omega$ $=8.8 \mathrm{eV}$ [left plot in Fig. 8(c)], we observe a strong localization of the field in the gap between the two particles, revealing a strong interaction between the spheres, whereas in the region near to the electron trajectory there is a weak confinement of the field. On the other hand, in the case of the planar surface plasmon mode at $\hbar \omega_{s}=10.68 \mathrm{eV}$, shown on the right-hand side of Fig. 8(c), the localization is intense nearby the electron trajectory, and disappears in between the particles.

The effect of placing a second sphere below the top particle can be clearly observed when we compare the total momentum transfer in the transverse direction for a singleparticle and for the dimer cases $(R=40 \mathrm{~nm})$, in Fig. 9. The effect is similar to the case of small particles (see Fig. 7), even though in this case there is no such large difference in the magnitude of the momentum transfer. A key point to understand this is that for large particles $(R=40 \mathrm{~nm})$ the fields produced by the electron are mainly high-order modes localized in the proximity of the electron trajectory when the electron passes near the particle, thus no significant charge density is generated in the other side of the big particle (cav- ity) and therefore diminishing the effect of the presence of a second particle.

In general, we can conclude that the effect of placing a particle close to another particle is to pull the top particle toward the bottom particle. This conclusion is based in the results of comparisons between the single-particle and the dimer cases (see Fig. 7 for small particles and Fig. 9 for large particles). As pointed out, the intensities of the momentum transfer present very different values between one single particle and dimer for the small particles case while it is of the same order of magnitude for large particles.

It is well known that the hybridization of the modes of single particles depends on the separation distance $d$, therefore this is a relevant parameter governing the excitation of surface modes in particle dimers. ${ }^{17,20}$ To show this effect, we consider the case of two big spherical particles of radius $R$ $=40 \mathrm{~nm}$ and modify the separation distance within the range $d=0.3-10 \mathrm{~nm}$, for three different electron impact parameters: $b=0.5,5$, and $40 \mathrm{~nm}$. We show the results for the momentum transfer to the top particle in a dimer along the $z$ direction (transverse direction) in Fig. 10, for these three different impact parameters, as a function of the separation distance between the particles, $d$. The impact parameter $b$ establishes the sign of the force for the single-particle case.

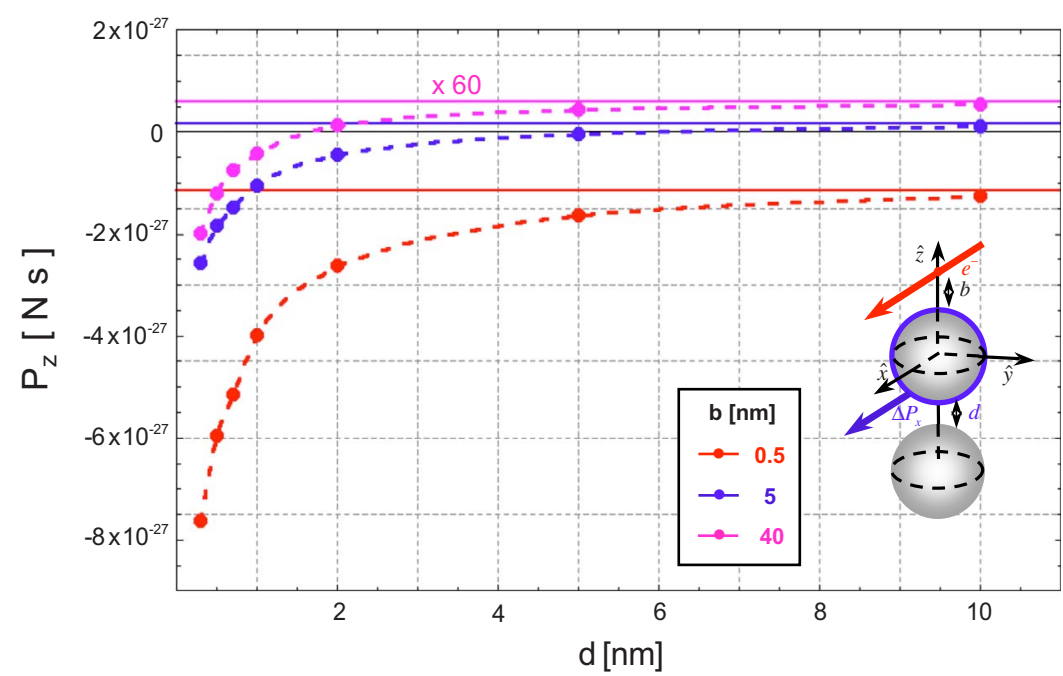

FIG. 10. (Color online) $z$ component of the momentum transfer from a fast electron to a top Drude-type spherical particle in a dimer configuration of identical particles of radius $R=40 \mathrm{~nm}$, as a function of the separation distance $d$ between the particles, for three different impact parameters: $b=0.5,5$, and $40 \mathrm{~nm}$. The $b=40 \mathrm{~nm}$ case is multiplied by a factor of 60 for a better appreciation. The horizontal line represents the singleparticle limit corresponding to each value of the impact parameter and dashed lines are a guide to the eyes. 
This limit has been represented as a horizontal line for each impact parameter considered in Fig. 10. As the separation distance between particles $d$ decreases, the momentum transferred to the top particle becomes more negative, i.e., more repulsion between the top particle and the electron is produced, pushing the top particle more intensely toward the bottom one (see red dashed lines in Fig. 10). In this way, it is possible even to switch the sign of the force by approaching a second particle (change from positive to negative values in Fig. 10). These results confirm the possibility to control the force induced at a particle by the combined design of electron impact parameter and separation distance between particles, as Fig. 10 demonstrates.

\section{B. Gold dimer}

We consider now the situation of a dimer made of gold nanoparticles, as a realistic and experimentally available material. We calculate the momentum transfer from the electron to the top particle in the dimer. We consider the geometry shown in Fig. 1(b) with two identical gold particles of radius $R=1 \mathrm{~nm}$ in vacuum and separated by a distance of $d$ $=0.25 \mathrm{~nm}$. We analyze several impact parameters of the electron beam in the range of $b=0.3-10 \mathrm{~nm}$. The plots for both $x$ (longitudinal) and $z$ (transverse) directions of the differential momentum transfer per unit frequency for the top particle are shown in Figs. 11(a) and 11(b), respectively. We observe that both $d P_{x} / d \omega$ and $d P_{z} / d \omega$ show a similar behavior to that in the case of a single gold particle [Figs. 5(a) and 5(b)], however a richer modal structure in the range of 0-20 $\mathrm{eV}$ can be appreciated in the spectrum for this dimer case, with noticeable differences in the intensity of the low-energy plasmon mode at $\hbar \omega \approx 2.5 \mathrm{eV}$. For the momentum transfer to the top particle in the $x$ direction as a function of the impact parameter $b$, similar trends can be observed for one single particle [inset in Fig. 5(a)] and for the dimer case [inset in Fig. 11(a)]. On the contrary, for the $z$ component there are evident differences in the intensity of the momentum transfer as a function of the impact parameter $b$ [inset in Fig. 11(b)], even though the dependence of $d P_{z} / d \omega$ on $\omega$ exhibits small differences compared with the single-particle case [see Fig. 5(b)]. For all impact parameters considered in the dimer case, the momentum transfer along the perpendicular direction to the electron trajectory on the top particles is always negative, that is, the top particle will tend to move toward the bottom particle in all cases for these external trajectories. In order to clarify the differences in the momentum transfer along the $z$ component between the single and dimer cases, we show in Fig. 12 the momentum transfer $P_{z}$ as a function of $b$ for both gold single particles and gold dimers. While for the single-particle case there is a maximum of attractive interaction between the particle and the electron at around $b=0.7 \mathrm{~nm}$, becoming repulsive for smaller impact parameters, in the case of the dimer, the interaction on the top particle is always repulsive with respect to the electron trajectory, pushing the top particle toward the bottom particle.

We also calculate near-field plots of the magnitude of the induced electric field around the particles, shown in Fig. 11(c), for two selected frequencies that present resonance peaks denoted by arrows in Fig. 11(b). For the lower energy plasmon mode $\hbar \omega=2.3 \mathrm{eV}$ [see left plot in Fig. 11(c)], we observe that the fields are concentrated both in the gap between the two particles (showing a strong interaction between the spheres) and in the region close to the electron trajectory (marked by an arrow). For very high-energy values as $\hbar \omega=38 \mathrm{eV}$ [see right plot in Fig. 11(c)], the field concentrates mainly in the zone closer to the electron trajectory and there is practically no field between the two particles, showing a weak interaction between the spheres.

\section{CONCLUSIONS}

We have studied the momentum transfer from a fast electron to a metallic particle, considering spherical particles made of two different materials: aluminum and gold. Two different configurations have been highlighted, a single particle and dimers, to explore how the presence of the second particle modifies the momentum transfer of the top particle, due to the generation of bonding dimer plasmons that localize strongly at the dimers cavity modifying the movement of the particle when the electron beam triggers out these modes. The relevant parameters in the problem: radii of the particle, impact parameter of the electron, and separation distance between particles in the dimer have been analyzed in detail and different behaviors on the motion of the particles have been obtained for different parameter ranges. We can conclude that, in general, the interaction between the particle and the electron is attractive for large values of the impact parameter (dipolarlike excitation) but the interaction becomes repulsive for close impact parameters (population of higher order multipolar excitations).

Comparing a single particle characterized by a Drudetype response with a gold particle, the momentum transfer along the electron trajectory shows practically the same trends for both cases and for all impact parameters considered, despite the fact that the dependence of the differential momentum transfer in the $x$ direction is completely different on going from Drude particles to $\mathrm{Au}$ particles. For the $z$ component (perpendicular to the electron trajectory), the momentum transfer as a function of the impact parameter displays a maximum of intensity of attractive momentum transfer (particle toward the electron) and for smaller impact parameters, the particle-electron interaction becomes repulsive. The behavior for both materials considered is similar so one can conclude that these are common features to all metallic particles. The maximum (attractive) intensity of the momentum transfer in the $z$ direction for both cases (Drudetype and gold particles) is located around the same value of the impact parameter and it is directly related with the size of the particle. However, the intensity of the transfer is around one order of magnitude bigger for the case of gold particles due to the contribution of high-energy excitations that contribute to the momentum transfer.

Generally speaking, the effect of placing a second particle in the proximity of a single metallic particle is to pull the top particle down toward the bottom particle for external electron trajectories on the top. We have clearly identified this 
(a)

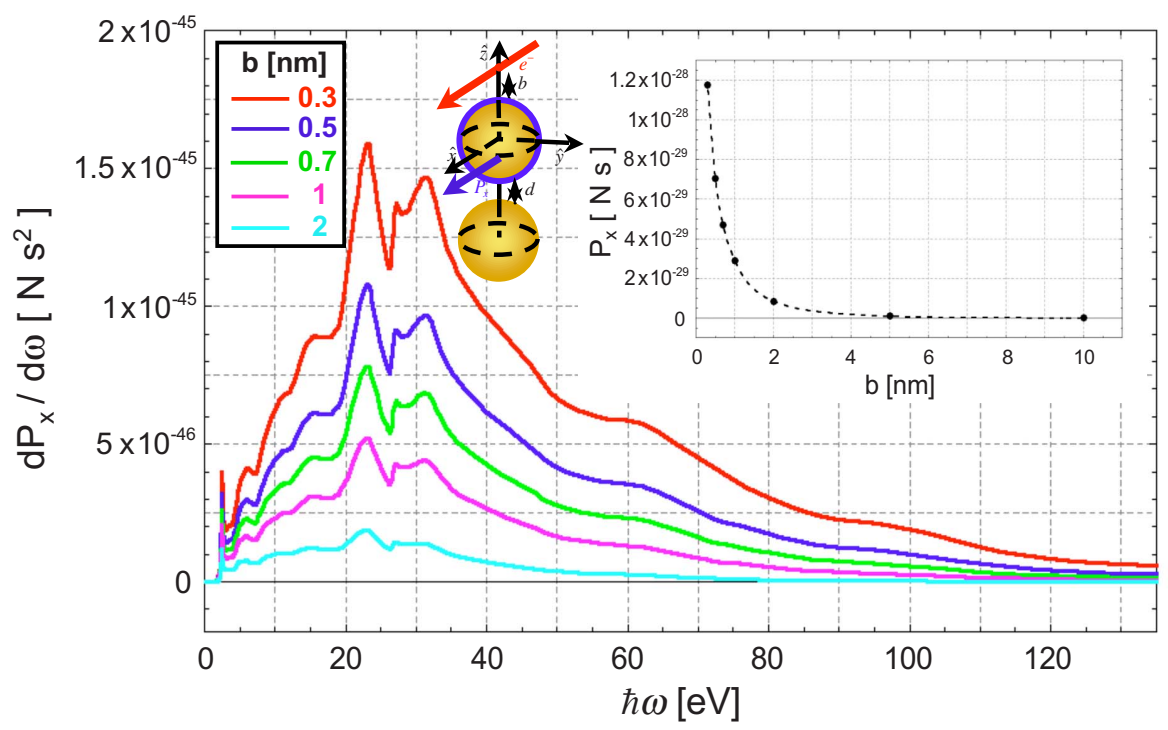

(b)

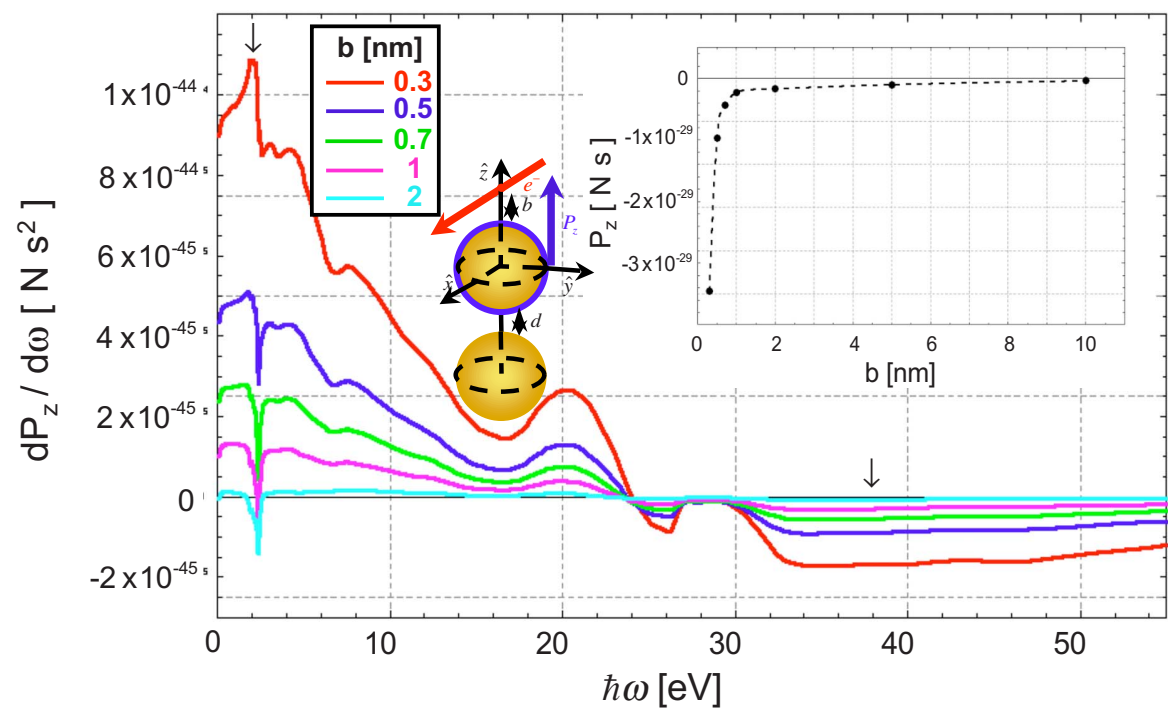

(c)
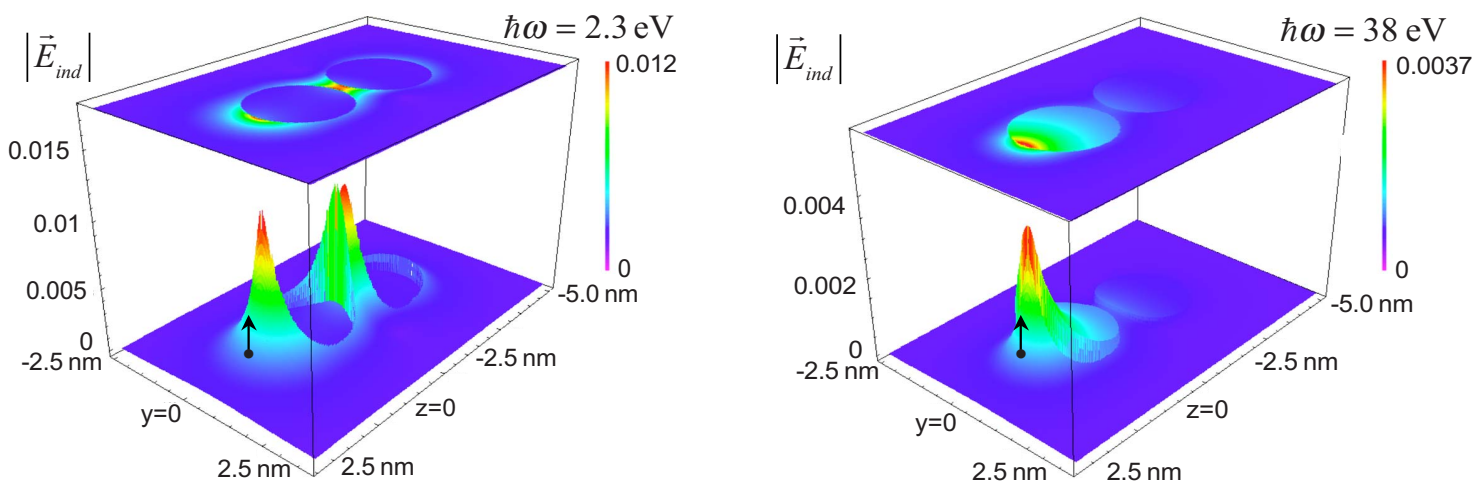

FIG. 11. (Color online) Frequency contribution to (a) $x$ and (b) $z$ components of the momentum transfer from the fast electron to a top gold spherical particle in a dimer configuration of identical particles of radius $R=1 \mathrm{~nm}$ separated by a distance $d=0.25 \mathrm{~nm}$, for different impact parameters $b$. Inset: (a) $x$ and (b) $z$ components of the momentum transfer to the top particle in the dimer as a function of the impact parameter $b$. The dashed line is a guide to the eyes. (c) 3D plot and 2D projection on top, of the magnitude of the induced electric field in the vicinity of a dimer of identical gold particles of radius $R=1 \mathrm{~nm}$, separated by a distance $d=0.25 \mathrm{~nm}$ with an impact parameter of the electron trajectory (indicated by a black arrow) of $b=0.3 \mathrm{~nm}$, for two selected values of the frequency denoted by arrows in (b). 




FIG. 12. (Color online) Comparison of total momentum transfer along $z$ direction as a function of the impact parameter $b$, between a single small $(R=1 \mathrm{~nm})$ gold particle and the top particle in a dimer of small $(R=1 \mathrm{~nm})$ gold identical particles separated by a distance $d=0.25 \mathrm{~nm}$. Dashed lines are a guide to the eyes. effect in Drude-type dimers for both small and large particles for all external impact parameters $b$ considered. This effect differs substantially from the single-particle case where the momentum transfer changes in sign as $b$ is modified. Similar trends have been obtained in gold dimers.

We have shown that the relevant parameters such as the size and material of the particles and the separation distance between particles in the dimer, determine the value of the impact parameter at which the transition between an attractive and repulsive interaction occurs. This effect allows for the pulling or pushing of nanoparticles with the fast electron beam on demand and opens the possibility of manipulating and assembling nanoscale objects. Further work can involve the movement of the probe to other symmetry positions: between the two spheres, at a similar impact parameter, or on the line of symmetry to the side of the spheres, for example. This situation might trigger out the excitation of symmetric plasmon modes that could push the spheres away from each other. The substrate effect should also be considered in a twofold manner: on one hand, the induced fields might be slightly modified due to the presence of the substrate even though the main trends will prevail, and on the other hand, cohesion forces between substrate and the particles might establish threshold values for the forces to be actually able to move the particles. Different substrates can present different thresholds that might be suitable for slowing down the motion of the particles allowing direct observation of the particle dynamics. As a summary, electron beams might be an extremely promising tool to manipulate and move some of the tiniest particles of the nanoworld on demand.

\section{ACKNOWLEDGMENTS}

Financial support from the Department of Industry of the Basque Government through the ETORTEK project inano, from the Spanish Ministerio de Ciencia e Innovación through Project No. FIS207-066711-C02-00, from the Consejo Nacional de Ciencia y tecnología (Mexico) through Project No. 82073, and from the U.S. NSF under Grant No. 0959905 are acknowledged.

\section{APPENDIX: ELECTROMAGNETIC FIELDS OF A FAST ELECTRON NEARBY A SINGLE SPHERICAL PARTICLE}

Following the formalism developed in Ref. 31 (where atomic units are used: $e=m=\hbar=1$ ) we can write Eqs. (12) and (13) in terms of a spherical basis. The components of the external electric field $\vec{E}_{\text {ext }}(\vec{r} ; \omega)$ in spherical coordinates $E_{\text {ext }}^{r}$, $E_{e x t}^{\theta}$, and $E_{e x t}^{\varphi}$ are given by

$$
\begin{gathered}
E_{e x t}^{r}(\vec{r} ; \omega)=\frac{-2 i \pi \omega}{c^{2} \gamma} \sum_{l=1}^{\infty} \frac{j_{l}(k r)}{k r} \sum_{m=-l}^{l} B_{l, m} K_{m}\left(\frac{\omega \xi}{v \gamma}\right) Y_{l, m}(\theta, \varphi), \\
E_{e x t}^{\theta}(\vec{r} ; \omega)=\frac{4 i \pi \omega v}{c^{3}} \sum_{l=1}^{\infty} j_{l}(k r) \sum_{m=-l}^{l} \frac{m^{2}}{l(l+1)} A_{l, m}^{+} K_{m}\left(\frac{\omega \xi}{v \gamma}\right) \frac{Y_{l, m}(\theta, \varphi)}{\sin \theta}+\frac{2 i \pi \omega}{c^{2} \gamma} \sum_{l=1}^{\infty}\left[(l+1) \frac{j_{l}(k r)}{k r}-j_{l+1}(k r)\right] \\
\times \sum_{m=-l}^{l} \frac{B_{l, m}}{l(l+1)} K_{m}\left(\frac{\omega \xi}{v \gamma}\right)\left[(l+1) \frac{\cos \theta}{\sin \theta} Y_{l, m}(\theta, \varphi)-\frac{(l-m+1)}{\sin \theta} \frac{\alpha_{l, m}}{\alpha_{l+1, m}} Y_{l+1, m}(\theta, \varphi)\right],
\end{gathered}
$$




$$
\begin{aligned}
E_{e x t}^{\varphi}(\vec{r} ; \omega)= & \frac{4 \pi \omega v}{c^{3}} \sum_{l=1}^{\infty} j_{l}(k r) \sum_{m=-l}^{l} \frac{m}{l(l+1)} A_{l, m}^{+} K_{m}\left(\frac{\omega \xi}{v \gamma}\right)\left[(l+1) \frac{\cos \theta}{\sin \theta} Y_{l, m}(\theta, \varphi)-\frac{(l-m+1)}{\sin \theta} \frac{\alpha_{l, m}}{\alpha_{l+1, m}} Y_{l+1, m}(\theta, \varphi)\right] \\
& +\frac{2 \pi \omega}{c^{2} \gamma} \sum_{l=1}^{\infty}\left[(l+1) \frac{j_{l}(k r)}{k r}-j_{l+1}(k r)\right] \sum_{m=-l}^{l} \frac{m}{l(l+1)} B_{l, m} K_{m}\left(\frac{\omega \xi}{v \gamma}\right) \frac{Y_{l, m}(\theta, \varphi)}{\sin \theta},
\end{aligned}
$$

while the components of the external magnetic field $\vec{H}_{\text {ext }}(\vec{r} ; \omega)$ are

$$
\begin{aligned}
& H_{e x t}^{r}(\vec{r} ; \omega)=\frac{-4 i \pi \omega v}{c^{3}} \sum_{l=1}^{\infty} \frac{j_{l}(k r)}{k r} \sum_{m=-l}^{l} m A_{l, m}^{+} K_{m}\left(\frac{\omega \xi}{v \gamma}\right) Y_{l, m}(\theta, \varphi), \\
& H_{\text {ext }}^{\theta}(\vec{r} ; \omega)=\frac{-2 i \pi \omega}{c^{2} \gamma} \sum_{l=1}^{\infty} j_{l}(k r) \sum_{m=-l}^{l} \frac{m}{l+1} B_{l, m} K_{m}\left(\frac{\omega \xi}{v \gamma}\right) \frac{Y_{l, m}(\theta, \varphi)}{\sin \theta} \\
& +\frac{4 i \pi \omega v}{c^{3}} \sum_{l=1}^{\infty}\left[(l+1) \frac{j_{l}(k r)}{k r}-j_{l+1}(k r)\right] \sum_{m=-l}^{l} \frac{m}{l(l+1)} A_{l, m}^{+} K_{m}\left(\frac{\omega \xi}{v \gamma}\right)\left[(l+1) \frac{\cos \theta}{\sin \theta} Y_{l, m}(\theta, \varphi)-\frac{(l-m+1)}{\sin \theta} \frac{\alpha_{l, m}}{\alpha_{l+1, m}(\theta, \varphi)}\right], \\
& H_{e x t}^{\varphi}(\vec{r} ; \omega)=\frac{4 \pi \omega v}{c^{3}} \sum_{l=1}^{\infty}\left[(l+1) \frac{j_{l}(k r)}{k r}-j_{l+1}(k r)\right] \sum_{m=-l}^{l} \frac{m^{2}}{l(l+1)} A_{l, m}^{+} K_{m}\left(\frac{\omega \xi}{v \gamma}\right) \frac{Y_{l, m}(\theta, \varphi)}{\sin \theta} \\
& -\frac{2 \pi \omega}{c^{2} \gamma} \sum_{l=1}^{\infty} j_{l}(k r) \sum_{m=-l}^{l} \frac{B_{l, m}}{l(l+1)} K_{m}\left(\frac{\omega \xi}{v \gamma}\right)\left[(l+1) \frac{\cos \theta}{\sin \theta} Y_{l, m}(\theta, \varphi)-\frac{(l-m+1)}{\sin \theta} \frac{\alpha_{l, m}}{\alpha_{l+1, m}} Y_{l+1, m}(\theta, \varphi)\right],
\end{aligned}
$$

where $c$ is the speed of light in vacuum, $i=\sqrt{-1}, v$ is the speed of the electron, $j_{l}(\rho)$ is the spherical Bessel function of first kind, $k=\omega / c$ is the magnitude of the wave vector in vacuum, $K_{m}$ is the modified Bessel function of order $m$, $\gamma=1 / \sqrt{1-(v / c)^{2}}, Y_{l, m}$ are the spherical harmonics, and $\xi$ is the distance from the origin to the beam position, i.e., $\xi=a$ $+b$, with $a$ the radius of the spherical particle and $b$ the impact parameter. The conventions for the special functions used here are defined in Ref. 30.

The coefficients $A_{l, m}^{+}$are given by ${ }^{31}$

$$
A_{l, m}^{+}=\frac{1}{\beta^{l+1}} \sum_{j=m}^{l} \frac{(i)^{l-j}(2 l+1) ! ! \alpha_{l, m}}{\gamma^{j} 2^{j}(l-j) ![(j-m / 2)] ![(j+m) / 2] !} I_{j, l-j}^{l, m},
$$

where

$$
\alpha_{l, m}=\sqrt{\frac{2 l+1}{4 \pi} \frac{(l-m) !}{(l+m) !}}
$$

and the numbers $I_{j, l-j}^{l, m}$ are calculated using the recurrence relation

$$
(l-m) I_{i_{1}, i_{2}}^{l, m}=(2 l-1) I_{i_{1}, i_{2+1}}^{l-1, m}-(l+m-1) I_{i_{1}, i_{2}}^{l-2, m},
$$

with the starting values being $I_{i_{1}, i_{2}}^{m-1, m}=0, I_{i_{1}, i_{2}}^{m-2, m}=0$, and

$$
I_{i_{1}, i_{2}}^{m, m}= \begin{cases}(-1)^{m}(2 m-1) ! B\left(\frac{i_{1}+m+2}{2}, \frac{i_{2}+1}{2}\right) & i_{2} \text { even } \\ 0 & i_{2} \text { odd }\end{cases}
$$

where $B$ is the beta function. ${ }^{30}$ The coefficients $B_{l, m}$ are given in terms of the coefficients $A_{l, m}$,

$$
B_{l, m}=A_{l, m+1}^{+} \sqrt{(l+m+1)(l-m)}-A_{l, m-1}^{+} \sqrt{(l-m+1)(l+m)} .
$$

The components of the induced electric field $\vec{E}_{\text {ind }}(\vec{r} ; \omega)$ are given by

$$
E_{\text {ind }}^{r}(\vec{r} ; \omega)=\frac{2 \pi \omega}{c^{2} \gamma} \sum_{l=1}^{\infty} t_{l}^{E} \frac{h_{l}^{(1)}(k r)}{k r} \sum_{m=-l}^{l} B_{l, m} K_{m}\left(\frac{\omega \xi}{v \gamma}\right) Y_{l, m}(\theta, \varphi),
$$




$$
\begin{aligned}
& E_{\text {ind }}^{\theta}(\vec{r} ; \omega)= \frac{-4 \pi \omega v}{c^{3}} \sum_{l=1}^{\infty} \frac{t_{l}^{M}}{l(l+1)} h_{l}^{(1)}(k r) \sum_{m=-l}^{l} m^{2} A_{l, m}^{+} K_{m}\left(\frac{\omega \xi}{v \gamma}\right) \frac{Y_{l, m}(\theta, \varphi)}{\sin \theta} \\
&-\frac{2 \pi \omega}{c^{2} \gamma} \sum_{l=1}^{\infty} \frac{t_{l}^{E}}{l(l+1)}\left[(l+1) \frac{h_{l}^{(1)}(k r)}{k r}-h_{l+1}^{(1)}(k r)\right] \sum_{m=-l}^{l} B_{l, m} K_{m}\left(\frac{\omega \xi}{v \gamma}\right) \\
& \times\left[(l+1) \frac{\cos \theta}{\sin \theta} Y_{l, m}(\theta, \varphi)-\frac{(l-m+1)}{\sin \theta} \frac{\alpha_{l, m}}{\alpha_{l+1, m}} Y_{l+1, m}(\theta, \varphi)\right], \\
& E_{\text {ind }}^{\varphi}(\vec{r} ; \omega)=\frac{4 \pi \omega v}{c^{3}} \sum_{l=1}^{\infty} \frac{t_{l}^{M}}{l(l+1)} h_{l}^{(1)}(k r) \sum_{m=-l}^{l} m A_{l, m}^{+}\left[(l+1) \frac{\cos \theta}{\sin \theta} Y_{l, m}(\theta, \varphi)-\frac{(l-m+1)}{\sin \theta} \frac{\alpha_{l, m}}{\alpha_{l+1, m}} Y_{l+1, m}(\theta, \varphi)\right] K_{m}\left(\frac{\omega \xi}{v \gamma}\right) \\
&+\frac{2 \pi \omega}{c^{2} \gamma} \sum_{l=1}^{\infty} \frac{t_{l}^{E}}{l(l+1)}\left[(l+1) \frac{h_{l}^{(1)}(k r)}{k r}-h_{l+1}^{(1)}(k r)\right] \sum_{m=-l}^{l} \frac{m}{\sin \theta} B_{l, m} K_{m}\left(\frac{\omega \xi}{v \gamma}\right) Y_{l, m}(\theta, \varphi),
\end{aligned}
$$

while for the induced magnetic field $\vec{H}_{\text {ind }}(\vec{r} ; \omega)$ are

$$
\begin{gathered}
H_{\text {ind }}^{r}(\vec{r} ; \omega)=\frac{4 \pi \omega v}{c^{3}} \sum_{l=1}^{\infty} t_{l}^{M} \frac{h_{l}^{(1)}(k r)}{k r} \sum_{m=-l}^{l} m A_{l, m}^{+} K_{m}\left(\frac{\omega \xi}{v \gamma}\right) Y_{l, m}(\theta, \varphi), \\
H_{\text {ind }}^{\theta}(\vec{r} ; \omega)=\frac{2 \pi \omega}{c^{2} \gamma} \sum_{l=1}^{\infty} \frac{t_{l}^{E}}{l(l+1)} h_{l}^{(1)}(k r) \sum_{m=-l}^{l} \frac{m}{\sin \theta} B_{l, m} K_{m}\left(\frac{\omega \xi}{v \gamma}\right) Y_{l, m}(\theta, \varphi) \\
-\frac{4 \pi \omega v}{c^{3}} \sum_{l=1}^{\infty} \frac{t_{l}^{M}}{l(l+1)}\left[(l+1) \frac{h_{l}^{(1)}(k r)}{k r}-h_{l+1}^{(1)}(k r)\right] \sum_{m=-l}^{l} m A_{l, m}^{+} K_{m}\left(\frac{\omega \xi}{v \gamma}\right) \\
\times\left[(l+1) \frac{\cos \theta}{\sin \theta} Y_{l, m}(\theta, \varphi)-\frac{(l-m+1)}{\sin \theta} \frac{\alpha_{l, m}}{\alpha_{l+1, m}} Y_{l+1, m}(\theta, \varphi)\right], \\
H_{\text {ind }}^{\varphi}(\vec{r} ; \omega)=\frac{-2 \pi \omega}{c^{2} \gamma} \sum_{l=1}^{\infty} \frac{t_{l}^{E}}{l(l+1)} h_{l}^{(1)}(k r) \sum_{m=-l}^{l} B_{l, m}\left[(l+1) \frac{\cos \theta}{\sin \theta} Y_{l, m}(\theta, \varphi)-\frac{(l-m+1)}{\sin \theta} \frac{\alpha_{l, m}}{\alpha_{l+1, m}} Y_{l+1, m}(\theta, \varphi)\right] K_{m}\left(\frac{\omega \xi}{v \gamma}\right) \\
+\frac{4 \pi \omega v}{c^{3}} \sum_{l=1}^{\infty} \frac{t_{l}^{M}}{l(l+1)}\left[(l+1) \frac{h_{l}^{(1)}(k r)}{k r}-h_{l+1}^{(1)}(k r)\right] \sum_{m=-l}^{l} \frac{m^{2}}{\sin \theta} A_{l, m}^{+} K_{m}\left(\frac{\omega \xi}{v \gamma}\right) Y_{l, m}(\theta, \varphi),
\end{gathered}
$$

where $h_{l}^{(1)}(\rho)$ is the spherical Hankel function. The dependence of the induced fields on the radius of the particle $a$ is given through the coefficients $t_{l}^{M}$ and $t_{l}^{E}$, which involve the spherical Bessel and Hankel functions

$$
\begin{gathered}
t_{l}^{M}=(-i) \frac{j_{l+1}\left(x_{0}\right) j_{l}\left(x_{i}\right)-N_{I} j_{l}\left(x_{0}\right) j_{l+1}\left(x_{i}\right)}{N_{I} j_{l+1}\left(x_{i}\right) h_{l}^{(1)}\left(x_{0}\right)-j_{l}\left(x_{i}\right) h_{l+1}^{(1)}\left(x_{0}\right)}, \\
t_{l}^{E}=(-i) \frac{(n+1)\left(\frac{1-N_{I}^{2}}{N_{I}}\right) j_{l}\left(x_{0}\right) j_{l}\left(x_{i}\right)-x_{0} j_{l}\left(x_{0}\right) j_{l+1}\left(x_{i}\right)+x_{i} j_{l}\left(x_{i}\right) j_{l+1}\left(x_{0}\right)}{(n+1)\left(\frac{N_{I}^{2}-1}{N_{I}}\right) j_{l}\left(x_{i}\right) h_{l}^{(1)}\left(x_{0}\right)-x_{i} j_{l}\left(x_{i}\right) h_{l+1}^{(1)}\left(x_{0}\right)+x_{0} j_{l+1}\left(x_{i}\right) h_{l}^{(1)}\left(x_{0}\right)}
\end{gathered}
$$

with $x_{0}=k a$ and $x_{i}=N_{I} k a$. Here $N_{I}=\sqrt{\varepsilon_{I}(\omega) / \varepsilon_{0}}$ is the index of refraction, with $\varepsilon_{I}$ and $\varepsilon_{0}$, the dielectric response function of the spherical particle and vacuum, respectively. 
*a.reyescoronado@gmail.com

†aizpurua@ehu.es

${ }^{1}$ P. E. Batson, Solid State Commun. 34, 477 (1980).

${ }^{2}$ P. E. Batson, Phys. Rev. Lett. 49, 936 (1982).

${ }^{3}$ P. E. Batson, Ultramicroscopy 9, 277 (1982).

${ }^{4}$ A. Howie and R. H. Milne, J. Microsc. 136, 279 (1984).

${ }^{5}$ A. Howie, Ultramicroscopy 11, 141 (1983).

${ }^{6}$ A. Howie and C. A. Walsh, Radiat. Eff. Defects Solids 117, 169 (1991).

${ }^{7}$ R. H. Ritchie, P. M. Echenique, F. Flores, and J. R. Manson, Radiat. Eff. Defects Solids 117, 163 (1991).

${ }^{8}$ D. Ugarte, C. Colliex, and P. Trebbia, Phys. Rev. B 45, 4332 (1992).

${ }^{9}$ J. Nelayah, M. Kociak, O. Stéfan, F. J. García de Abajo, M. Tencé, L. Henrard, D. Taverna, I. Pastoriza-Santos, L. M. Liz-Marzán, and C. Colliex, Nat. Phys. 3, 348 (2007).

${ }^{10}$ A. L. Koh, K. Bao, I. Khan, W. E. Smith, G. Kothleitner, P. Nordlander, S. A. Maier, and D. W. McComb, ACS Nano 3, 3015 (2009).

${ }^{11}$ J. Nelayah, M. Kociak, O. Stéphan, N. Geuquet, L. Henrard, F. J. García de Abajo, I. Pastoriza-Santos, L. M. Liz-Marzán, and C. Colliex, Nano Lett. 10, 902 (2010).

${ }^{12}$ F. J. García de Abajo, Rev. Mod. Phys. 82, 209 (2010).

${ }^{13}$ P. E. Batson, Microsc. Microanal. 14, 89 (2008).

${ }^{14}$ R. H. Ritchie, Phys. Rev. 106, 874 (1957).

${ }^{15}$ T. L. Ferrell and P. M. Echenique, Phys. Rev. Lett. 55, 1526 (1985).

${ }^{16}$ F. J. García de Abajo, Phys. Rev. B 70, 115422 (2004).

${ }^{17}$ P. Nordlander, C. Oubre, E. Prodan, K. Li, and M. I. Stockman, Nano Lett. 4, 899 (2004).

${ }^{18}$ N. Zabala, A. Rivacoba, and P. M. Echenique, Phys. Rev. B 56, 7623 (1997)

${ }^{19}$ M. Schmeits and L. Dambly, Phys. Rev. B 44, 12706 (1991).

${ }^{20}$ I. Romero, J. Aizpurua, G. W. Bryant, and F. J. García de Abajo, Opt. Express 14, 9988 (2006).

${ }^{21}$ E. Prodan, C. Radloff, N. J. Halas, and P. Nordlander, Science 302, 419 (2003).
${ }^{22}$ H. Xu, J. Aizpurua, M. Käll, and P. Apell, Phys. Rev. E 62, 4318 (2000).

${ }^{23}$ A. J. Hallock, P. L. Redmond, and L. E. Brus, Proc. Natl. Acad. Sci. U.S.A. 102, 1280 (2005).

${ }^{24}$ H. Xu and M. Käll, Phys. Rev. Lett. 89, 246802 (2002).

${ }^{25}$ V. D. Miljković, T. Pakizeh, B. Sepulveda, P. Johansson, and M. Käll, J. Phys. Chem. C 114, 7472 (2010).

${ }^{26}$ A. Rivacoba, N. Zabala, and J. Aizpurua, Prog. Surf. Sci. 65, 1 (2000).

${ }^{27}$ J. D. Jackson, Classical Electrodynamics, 3rd ed. (Wiley, New York, 1999).

${ }^{28}$ To obtain the integral in Eq. (1), $\vec{P}_{E M}$ is evaluated at times $t=-\infty$ and $t=\infty$, which correspond to times when the position of the electron is infinitely far from the evaluation region $V$ contained by the surface $S$, thus the electromagnetic field inside the volume $V$ is negligible.

${ }^{29}$ E. D. Palik, Handbook of Optical Constants of Solids (Academic Press, Orlando, 1985).

${ }^{30}$ M. Abramowitz and I. A. Stegun, Handbook of Mathematical Functions, 9th ed. (Dover, New York, 1972).

${ }^{31}$ F. J. García de Abajo, Phys. Rev. B 59, 3095 (1999).

${ }^{32}$ In the numerical evaluation of the surface integral a spherical grid surrounding the spherical particle is used with a large number of grid points to obtain convergence. The radius of the spherical grid was around one hundredth larger than the radius of the metallic particle. The final results of the surface integral show a no dependence on the radius of the spherical grid.

${ }^{33}$ J. Aizpurua, A. Rivacoba, and S. P. Apell, Phys. Rev. B 54, 2901 (1996).

${ }^{34}$ J. Aizpurua, A. Howie, and F. J. García de Abajo, Phys. Rev. B 60, 11149 (1999).

${ }^{35}$ A. Rivacoba and P. M. Echenique, Ultramicroscopy 26, 389 (1988).

${ }^{36}$ F. J. García de Abajo and J. Aizpurua, Phys. Rev. B 56, 15873 (1997).

${ }^{37}$ F. J. García de Abajo and A. Howie, Phys. Rev. B 65, 115418 (2002). 